\title{
Optical absorption and conductivity in quasi-two-dimensional crystals from first principles: Application to graphene
}

\author{
Dino Novko, ${ }^{1}$ Marijan Šunjić, ${ }^{1,2}$ and Vito Despoja ${ }^{1,2,3}$ \\ ${ }^{1}$ Donostia International Physics Center (DIPC), P. Manuel de Lardizabal, 20018 San Sebastian, Basque Country, Spain \\ ${ }^{2}$ Department of Physics, University of Zagreb, Bijenička 32, HR-10000 Zagreb, Croatia \\ ${ }^{3}$ Universidad del Pais Vasco, Centro de Fisica de Materiales CSIC-UPV/EHU-MPC, Av. Tolosa 72, E-20018 San Sebastian, Spain \\ (Received 11 December 2015; revised manuscript received 5 February 2016; published 8 March 2016)
}

\begin{abstract}
This paper gives a theoretical formulation of the electromagnetic response of the quasi-two-dimensional crystals suitable for investigation of optical activity and polariton modes. The response to external electromagnetic field is described by current-current response tensor $\Pi_{\mu \nu}$ calculated by solving the Dyson equation in the random phase approximation, where current-current interaction is mediated by the photon propagator $D_{\mu \nu}$. The irreducible current-current response tensor $\Pi_{\mu \nu}^{0}$ is calculated from the ab initio Kohn-Sham orbitals. The accuracy of $\Pi_{\mu \nu}^{0}$ is tested in the long-wavelength limit where it gives correct Drude dielectric function and conductivity. The theory is applied to the calculation of optical absorption and conductivity in pristine and doped single-layer graphene and successfully compared with previous calculations and measurements.
\end{abstract}

DOI: 10.1103/PhysRevB.93.125413

\section{INTRODUCTION}

Understanding the interaction between light and electrons in a crystal has always been an attractive topic, and its extensive study led to the realization of many devices, such as lasers, semiconducting solar cells, or LED diodes. More recently, it led to new discoveries, such as sub-wavelength light transmission [1], waveguiding [2], hybrid solid state/organic solar cells [3], etc. There are many theoretical models which successfully deal with these phenomena, mostly based on solving Maxwell's equations at the boundaries of the crystals of different shapes [4] and different dielectric properties, calculated at different levels of approximations, e.g., within the Drude dielectric model $[5,6]$, or from first principles and beyond the random phase approximation (RPA) [7].

However, what is still missing is a theoretical approach where the interaction between light and crystal electrons would be calculated fully microscopically, so that the electronic structure is calculated using ab initio methods (usually in the simplified tight-binding or subband models [8-12]), the polarizability of the system is described by the current-current response tensor (usually by the density-density response function [13]), and where the electron-electron interaction is mediated by photons (usually described by instantaneous Coulomb interaction). Inclusion of these effects could be crucial if one wanted to explore new optically active (radiative) modes or self-sustainable electromagnetic modes (polaritons) in crystals.

The aim of this paper is to give a theoretical formulation of the interaction between electromagnetic field and electronic excitations in quasi-two-dimensional (q2D) crystals (consisting of one or few atomic layers), suitable for investigation of optically active electronic modes and polaritons. This formulation was partially developed in Ref. [14] where it was applied to investigate electromagnetic modes in the jellium metallic slab. Here, this formulation is extended to the case where the ground-state electronic structure is calculated from first principles. To test the theory, we calculate the optical absorption and conductivity in the self-standing graphene monolayer and compare our results with the recent experimental and theoretical works [15-24].

Optical properties of graphene have already been extensively investigated, both from the experimental and theoretical viewpoints. Apart from the above-mentioned works, the information about optical activity of $\pi$ or $\pi+\sigma$ plasmons or single-particle excitations was also extracted from electron energy loss (EELS) experiments and corresponding calculations [25-31]. In some cases, the dispersion relations of 2D polaritons and conductivity in doped graphene are calculated using RPA density-density response function [30,32-34] or using additionally a phenomenological relaxation-time approximation (RTA) to account for the damping effects [6,35]. In Refs. [35,36] optical properties and conductivity in graphene are investigated at a high level of accuracy, beyond RPA, however, the orbital and band structures are described within the tight-binding approximation (TBA). On the other hand, in Ref. [15] optical properties of graphene are calculated from first principles including quasiparticle corrections and solving Bethe-Salpeter equation (so-called GW-BSE scheme) for the polarizability tensor. This GW-BSE scheme includes excitonic effects properly, resulting in a nice agreement of ultraviolet (UV) active $\pi \rightarrow \pi^{*}$ peak with the experimental measurements, while our RPA theory underestimates this experimental value.

It is important to specify the main advantages of the present theory. First of all, the tensorial character of the response allows the resolution of the polarization and incident angle. In other words, we are able to analyze transverse electric, $\mathbf{s}(\mathrm{TE})$, and transverse magnetic, $\mathbf{p}(\mathrm{TM})$, modes separately, as well as the incident angle dependence of the response they produce. The inclusion of the retardation effects can strongly influence the dispersion relations of plasmon-polariton (PP) modes in metallic and doped semiconductor systems. One example is PP dispersion relation in doped graphene, as will be presented later. Also, if retardation effects are not included, the $\mathbf{s}(\mathrm{TE})$ mode disappears. However, $\mathbf{s}(\mathrm{TE})$ mode in doped graphene shows very interesting collective character [37-39]. Another advantage is computationally cheap but accurate calculation 
of the direct current (dc) and infrared (IR) optical conductivity, through which we can also observe electromagnetic collective modes. For example, combining paramagnetic and diamagnetic contributions of current-current response tensor into a single contribution using the charge continuity equation the computational cost is reduced, due to lower demand on the number of unoccupied bands in the new current-current expression with respect to the former one. This current-current response tensor can also be generalized in order to include the relaxation processes beyond RTA (electron-phonon, electronimpurity, and electron-electron scatterings). For some cases, this extension can be done by replacing the damping constant $i \eta$ with the so-called memory function $M(\mathbf{Q}, \omega)$, which can be calculated by using the many-body techniques [35]. If the corresponding single-particle self-energy terms and vertex corrections are considered on the same footing, then this could predict the accurate dc conductivity in the layered materials or nowadays intriguing ballistic regime in graphene [40].

It is worth mentioning, due to recent interest [41-44], that methodology presented in this paper can be adopted to obtain the charge-charge response function $\chi(\mathbf{Q}, \omega)$ and the dielectric function $\varepsilon(\mathbf{Q}, \omega)$ of $\mathrm{q} 2 \mathrm{D}$ material by connecting the former with current-current correlation function $\Pi_{\mu \nu}(\mathbf{Q}, \omega)$ using the gauge invariance and the conservation of local charge density $[35,45]$. Numerically, this can be very convenient for obtaining $\varepsilon(\mathbf{Q}, \omega)$ within RPA response because the $\mathbf{Q}^{2}$ divergence of the bare Coulomb interaction is automatically canceled due to $\mathbf{Q}$ prefactor in current vertex function and the special care for $\mathbf{Q}=0$ case [46] is not needed.

In Sec. II A, we first present the general formulation of the problem, description of the system, and the derivation of the Dyson equation for the screened current-current response tensor $\Pi_{\mu \nu}$. In Sec. II B, we formulate the Dyson equation for a specific geometry of the system in terms of Kohn-Sham (KS) electronic wave functions. In Sec. II C, the expressions for optical absorption and conductivity are given in terms of the tensor $\Pi_{\mu \nu}$. During $a b$ initio calculation of nonlocal paramagnetic and local diamagnetic terms in $\Pi_{\mu \nu}$ certain numerical problems arise which are discussed in Sec. IID and resolved using an alternative expression for the current-current response tensor. In Sec. II E, we prove that alternative expression of $\Pi_{\mu \nu}^{0}$ in the long-wavelength limit leads to the Drude dielectric function and conductivity. In Sec. III, we apply the developed methodology to calculate the optical absorption spectrum and conductivity in doped and pristine graphene and compare it with recent experimental and theoretical results. Section III A gives details of the computational procedure. We use density functional theory (DFT) ground-state calculation to get the crystal KS orbitals $\phi_{n \mathbf{K}}$ and band structure $E_{n \mathbf{K}}$. For the ground-state calculation we use the plane-wave self-consistent field (PWSCF) method which means that our crystal should be $3 \mathrm{D}$ periodic. Here, the superlattice consists of periodically repeated supercells containing several atomic layers (crystal slab). We also explain how to avoid the effect of interaction with the neighboring supercells. In the second stage of the calculation, we solve the Dyson equation for current-current response tensor $\Pi_{\mu \nu}$ where irreducible current-current response tensor $\Pi_{\mu \nu}^{0}$ enters and electromagnetic interaction is mediated by the photonic propagator $D_{\mu \nu}$ [14]. Here, we restrict our calculations to RPA where irreducible current-current response tensor $\Pi_{\mu \nu}^{0}$ can be obtained directly from the crystal KS states. A problem arises from the fact that we want to investigate optical properties of single q2D crystal slab, while electronic structure is calculated for the entire 3D superlattice. This problem can not be solved simply by increasing the vertical separation between slabs because now each of them radiates electromagnetic field which spreads across the entire space and interaction between slabs is unavoidable. We solve this by cutting off the vertical range of the photon propagator $D_{\mu \nu}$ and allowing propagation only within one $\mathrm{q} 2 \mathrm{D}$ crystal slab. This procedure allows smaller vertical distances between adjacent slabs (enough to avoid the overlap between their charge densities) while still canceling the interaction between them. Similar method is successfully utilized for calculation of EELS spectrum in graphene [30,31]. In Sec. III B, we present and discuss in detail results for optical absorption spectra in pristine and doped graphene. In Sec. IV, we present the conclusion.

\section{FORMULATION OF THE PROBLEM}

\section{A. Derivation of the current-current response tensor}

In this section, we will first derive the Dyson equation for the screened current-current response tensor in the q2D crystal consisting of one or few atomic layers. We consider independent electrons which move in a local (DFT) crystal potential and interact with the electromagnetic field described by the vector potential operator $\mathbf{A}(\mathbf{r}, t)$. Then, the Hamiltonian of the system can be written as

$$
H=H_{0}^{e}+H_{0}^{\mathrm{EM}}+V^{\mathrm{in}},
$$

where

$$
H_{0}^{e}=\sum_{\mathbf{K}, n} E_{n \mathbf{K}} c_{n \mathbf{K}}^{\dagger} c_{n \mathbf{K}}
$$

describes noninteracting electrons in some local potential. Here, $c_{n \mathbf{K}}^{\dagger}$ is the creation operator of an electron in the Bloch state $\{n, \mathbf{K}\}$, with the wave function $\phi_{n \mathbf{K}}(\mathbf{r})$ and energy $E_{n \mathbf{K}}$, where $n$ is the band index and $\mathbf{K}$ is the electron wave vector in the plane parallel to crystal layers. $H_{0}^{\mathrm{EM}}$ is the Hamiltonian of free electromagnetic field. In the $\Phi=0$ gauge, the interacting Hamiltonian

$$
V^{\text {in }}=V^{p}+V^{d}
$$

consists of the paramagnetic part

$$
V^{p}=-\frac{1}{c} \int d \mathbf{r} \mathbf{j}(\mathbf{r}) \mathbf{A}(\mathbf{r})
$$

and the diamagnetic part

$$
V^{d}=\frac{e^{2}}{2 m c^{2}} \int d \mathbf{r} \rho(\mathbf{r}) \mathbf{A}^{2}(\mathbf{r}) .
$$

Here, the electron current operator is

$$
\mathbf{j}(\mathbf{r})=\frac{e \hbar}{2 i m}\left\{\psi^{\dagger}(\mathbf{r}) \nabla \psi(\mathbf{r})-\left[\nabla \psi^{\dagger}(\mathbf{r})\right] \psi(\mathbf{r})\right\},
$$

electron density operator is

$$
\rho(\mathbf{r})=\psi^{\dagger}(\mathbf{r}) \psi(\mathbf{r})
$$




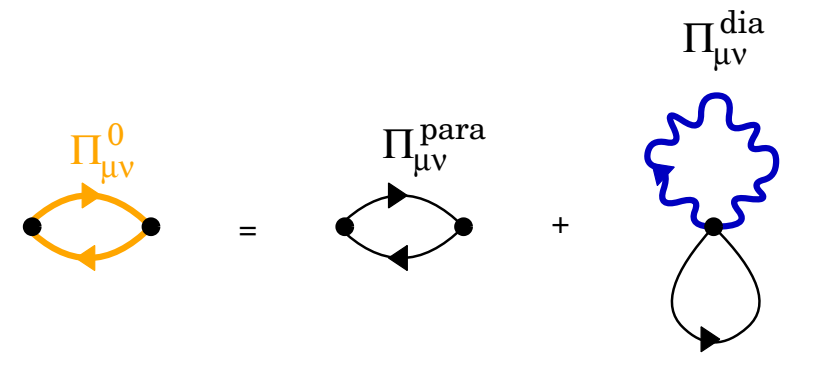

FIG. 1. Diagrammatic representation of the noninteracting current-current response tensor. The first term of the right-hand side represents the paramagnetic term (10) and the second term represents the diamagnetic term (11). Thin solid line represents one particle (hole) propagator and the wavy line represents the free-photon propagator $\mathbf{D}^{0}$.

and electron field operators are

$$
\psi(\mathbf{r})=\sum_{n, \mathbf{K}} \phi_{n \mathbf{K}}(\mathbf{r}) c_{n \mathbf{K}} .
$$

The key quantity that will give the physical properties (e.g., optical properties) of the system of electrons interacting with the "internal" electromagnetic field (electron-electron interaction mediated by photons) will be the current-current response tensor $\Pi_{\mu \nu}\left(\mathbf{r}, \mathbf{r}^{\prime}, t, t^{\prime}\right)$, which in the zeroth order of the perturbation expansion over interaction $V^{\text {in }}$ contains two terms:

$$
\Pi_{\mu \nu}^{0}=\Pi_{\mu \nu}^{\mathrm{para}}+\Pi_{\mu \nu}^{\mathrm{dia}} .
$$

Here, the paramagnetic term is

$$
\Pi_{\mu \nu}^{\mathrm{para}}\left(\mathbf{r}, \mathbf{r}^{\prime}, t, t^{\prime}\right)=\frac{i}{\hbar c} \theta\left(t-t^{\prime}\right)\left\langle\Psi_{e}^{0}\left|\left[j_{\mu}(\mathbf{r}, t), j_{\nu}\left(\mathbf{r}^{\prime}, t^{\prime}\right)\right]_{-}\right| \Psi_{e}^{0}\right\rangle
$$

and $\left|\Psi_{e}^{0}\right\rangle$ is the ground state of the Hamiltonian $H_{0}^{e}$. The diamagnetic term is

$$
\Pi_{\mu \nu}^{\mathrm{dia}}\left(\mathbf{r}, \mathbf{r}^{\prime}, t, t^{\prime}\right)=-\frac{e^{2}}{m c} n(\mathbf{r}) \delta\left(t-t^{\prime}\right) \delta\left(\mathbf{r}-\mathbf{r}^{\prime}\right) \delta_{\mu \nu},
$$

where $n(\mathbf{r})=\left\langle\Psi_{e}^{0}|\rho(\mathbf{r})| \Psi_{e}^{0}\right\rangle$ represents the ground-state electron density. In the lowest order, these two terms in the expansion of the irreducible current-current response tensor are represented diagrammatically in Fig. 1. Next step is to provide perturbation expansion for the tensor $\Pi$ which now includes the higher-order terms in the interaction $V^{\text {in }}$. If we restrict our consideration within RPA, the perturbation expansion of the current-current response tensor (9) becomes

$$
\begin{aligned}
\Pi= & \Pi_{0}+\Pi_{0} \otimes \mathbf{D}_{0} \otimes \Pi_{0} \\
& +\Pi_{0} \otimes \mathbf{D}_{0} \otimes \Pi_{0} \otimes \mathbf{D}_{0} \otimes \Pi_{0}+\ldots,
\end{aligned}
$$

where the symbol $\otimes$ denotes the convolution or integration over spatial and time variables $(\mathbf{r}, t)$ in addition to the matrix multiplication over indices $\mu=x, y, z$. Also for clarity we omit to write spatial and time variables. From the expansion (12) it is obvious that the "screened" current-current response tensor can be calculated by solving the Dyson equation

$$
\Pi=\Pi_{0}+\Pi_{0} \otimes \mathbf{D}_{0} \otimes \Pi,
$$

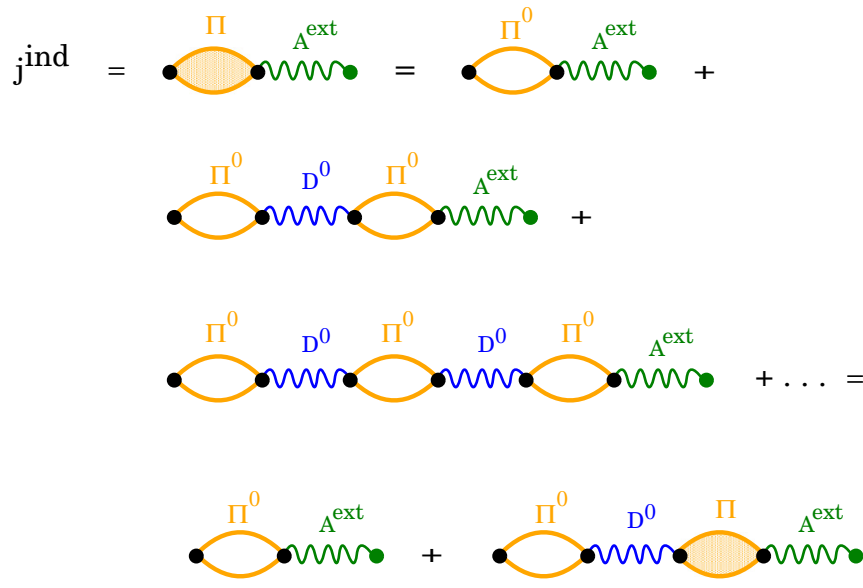

FIG. 2. Diagrammatic expansion of the induced current within RPA (16). $\Pi^{0}$ represents noninteracting current-current response tensor (9), $\Pi$ interacting current-current response tensor within RPA (13), and $D^{0}$ free-photon propagator (14)

where the only inputs are the noninteracting current-current response tensor $\Pi_{0}$ given by (9) and the retarded free-photon propagator given by

$$
\begin{aligned}
& D_{\mu \nu}^{0}\left(\mathbf{r}, \mathbf{r}^{\prime}, t, t^{\prime}\right) \\
& \quad=\frac{i}{\hbar c} \theta\left(t-t^{\prime}\right)\left\langle\Psi_{\mathrm{EM}}^{0}\left|\left[A_{\mu}^{0}(\mathbf{r}, t), A_{\nu}^{0}\left(\mathbf{r}^{\prime}, t^{\prime}\right)\right]_{-}\right| \Psi_{\mathrm{EM}}^{0}\right\rangle,
\end{aligned}
$$

where $\Psi_{\mathrm{EM}}^{0}$ is the photon vacuum (ground state of $H_{0}^{\mathrm{EM}}$ ), and the operator $A_{\mu}^{0}$ is defined as

$$
A_{\mu}^{0}(\mathbf{r}, t)=e^{i H_{0}^{\mathrm{EM}} t} A_{\mu}(\mathbf{r}) e^{-i H_{0}^{\mathrm{EM}} t} .
$$

The perturbation expansion of the current-current response tensor $\Pi$ is diagrammatically presented in Fig. 2, where the green wavy line represents the external field $\mathbf{A}^{\text {ext }}$, that induces current fluctuations in the crystal. Blue wavy lines represent the propagator of electromagnetic field $\mathbf{D}^{0}$, that mediates electromagnetic interaction within the crystal. It should be mentioned here that this expansion actually goes beyond RPA. Namely, current-current response function (9) can be calculated by means of single-particle Green's functions. On the other hand, electron-electron interaction is partially (depending on the DFT approximation used) included in the Bloch states used to construct the single-particle Green's function. Therefore, the electron-electron interaction is included already in the lowest order of the expansion (12) in the form of self-energy corrections to the irreducible polarizability $\Pi^{0}$.

The response tensor $\Pi_{\mu \nu}$ contains information about spectroscopic properties of the system (single-particle or collective electromagnetic modes in the system) or information about the response to the external electromagnetic field. Suppose that the crystal is exposed to the external (classical) electromagnetic field described by the vector potential $\mathbf{A}^{\mathrm{ext}}(\mathbf{r}, t)$. In this case, the total Hamiltonian (1) gets an additional term $V^{\text {ext }}$ which has the form analogous to (3)-(5) except that now A should be replaced by $\mathbf{A}^{\text {ext }}$. If $V^{\text {ext }}$ is a small perturbation, it is sufficient to keep only the term linear in $\mathbf{A}^{\text {ext }}$. In this case, the current 
induced by the external field becomes

$$
j_{\mu}^{\text {ind }}(\mathbf{r}, t)=\sum_{\nu} \int d \mathbf{r}_{1}, d t_{1} \Pi_{\mu \nu}\left(\mathbf{r}, \mathbf{r}_{1}, t-t_{1}\right) A_{\nu}^{\mathrm{ext}}\left(\mathbf{r}_{1}, t_{1}\right),
$$

as shown schematically in Fig. 2. The induced charge density fluctuations are similarly given by

$$
\rho^{\text {ind }}(\mathbf{r}, t)=\sum_{\mu} \int d \mathbf{r}_{1}, d t_{1} \Pi_{0 \mu}\left(\mathbf{r}, \mathbf{r}_{1}, t-t_{1}\right) A_{\mu}^{\mathrm{ext}}\left(\mathbf{r}_{1}, t_{1}\right),
$$

where we introduce the density-current response function which is (in the lowest order in expansion over $V^{\text {in }}$ ) given by

$$
\Pi_{0 v}^{0}\left(\mathbf{r}, \mathbf{r}^{\prime}, t, t^{\prime}\right)=\frac{i}{\hbar c} \theta\left(t-t^{\prime}\right)\left\langle\Psi_{e}^{0}\left|\left[\rho(\mathbf{r}, t), j_{v}^{\text {para }}\left(\mathbf{r}^{\prime}, t^{\prime}\right)\right]_{-}\right| \Psi_{e}^{0}\right\rangle
$$

Induced current and density fluctuations are connected by the continuity equation

$$
\nabla \mathbf{j}^{\text {ind }}+\frac{\partial}{\partial t} \rho^{\text {ind }}=0
$$

\section{B. Calculation of the screened current-current response tensor}

First, we shall explain how to solve the Dyson equation (12) so that the electromagnetic interaction between crystal slabs in the superlattice is avoided. Then, we shall exploit the symmetry of the system which leads to the conservation of the $\mathbf{Q}$ vector parallel to the surface and also enables division into $\mathbf{s}$ and $\mathbf{p}$ polarizations.

The plane-wave method implies 3D periodicity, so our system consists of periodically repeated crystal slabs, while the current-current response function $\Pi_{\mu \nu}^{0}$ is a periodic function in $z$ direction and it spreads over all space. The 3D character of $\Pi_{\mu \nu}^{0}$ enables the full Fourier transformation of the Dyson equation (12) and the photon propagator $D_{\mu \nu}^{0}$ becomes a diagonal matrix. However, due to long-range character of $D_{\mu \nu}^{0}$, the interaction between adjacent crystal slabs is possible, even if they are infinitely separated. The direct solution of such Dyson-matrix equation would lead to the screened $\Pi_{\mu \nu}$ which includes effects of the coupling between supercells. The main idea how to avoid this problem is to suppose that the supercells are not periodically repeated in the perpendicular $z$ direction, but that there is just one crystal slab in the system, which is restricted to the region $-L / 2<z<L / 2$, as sketched in Figs. 3(b) and 3(c). This implies that $\Pi_{\mu \nu}^{0}$ consists of just one term in the $z$ direction and all remaining terms are zero. So, even if the photon propagator $D_{\mu \nu}^{0}$ still propagates interaction all over the space it actually couples only to the charge or current fluctuations in the region $-L / 2<z<L / 2$. Solution of the corresponding Dyson equation (12) leads to $\Pi_{\mu \nu}$ which describes electromagnetic properties of the single isolated slab. The method how to solve such Dyson equation is described in the following.

The main physical quantities of our system (wave functions, charge density, etc.) are nonzero only in the region $-L / 2<$ $z<L / 2$, so the periodicity is broken in the $z$ direction. However, it remains in the $x y$ plane so it is possible to perform the Fourier transform of the Dyson equation (13) just in the

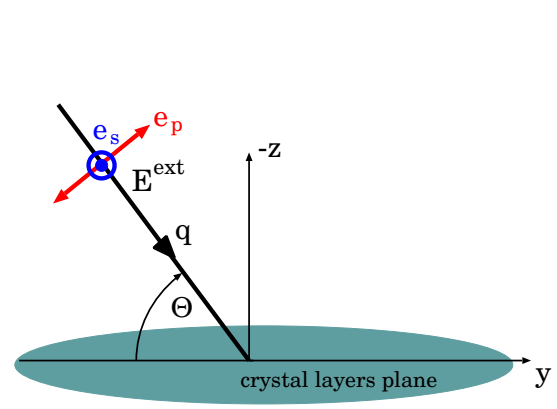

(a)
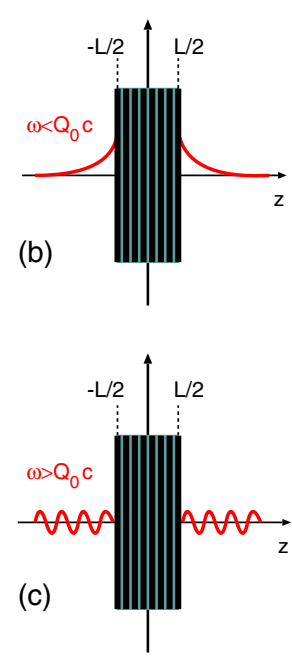

FIG. 3. Geometry of the system. (a) The orientation of the crystal layers and incident electromagnetic field. (b) Evanescent character of the incident electromagnetic field for $\omega<$ Qc. (c) Radiative character of the incident electromagnetic field for $\omega>$ Qc. Layered crystal electronic density is restricted in the region $-\frac{L}{2}<z<\frac{L}{2}$.

xy plane:

$$
\begin{aligned}
\Pi_{\mathbf{G}_{\|}, \mathbf{G}_{\|}^{\prime}}\left(\mathbf{Q}, \omega, z, z^{\prime}\right)= & \Pi_{\mathbf{G}_{\|}, \mathbf{G}_{\|}^{\prime}}^{0}\left(\mathbf{Q}, \omega, z, z^{\prime}\right) \\
& +\sum_{\mathbf{G}_{\| 1}, \mathbf{G}_{\| 2}} \int_{-\frac{L}{2}}^{\frac{L}{2}} d z_{1} d z_{2} \Pi_{\mathbf{G}_{\|}, \mathbf{G}_{\| 1}}^{0}\left(\mathbf{Q}, \omega, z, z_{1}\right) \\
& \times \mathbf{D}_{\mathbf{G}_{\| 1}^{0}, \mathbf{G}_{\| 2}}^{0}\left(\mathbf{Q}, \omega, z_{1}, z_{2}\right) \Pi_{\mathbf{G}_{\| 2}, \mathbf{G}_{\|}^{\prime}}\left(\mathbf{Q}, \omega, z_{2}, z^{\prime}\right),
\end{aligned}
$$

where $\mathbf{G}_{\|}=\left(G_{x}, G_{y}\right)$ are 2D reciprocal lattice vectors. In (20), we also simultaneously performed the Fourier transform in $\omega$ space. The Fourier transform of the free-photon propagator (14) is given by [14]

$$
\mathbf{D}_{\mathbf{G}_{\|}, \mathbf{G}_{\|}^{\prime}}^{0}\left(\mathbf{Q}, \omega, z, z^{\prime}\right)=\mathbf{D}^{0}\left(\mathbf{Q}+\mathbf{G}_{\|}, \omega, z, z^{\prime}\right) \delta_{\mathbf{G}_{\|}, \mathbf{G}_{\|}^{\prime}},
$$

where [47]

$$
\begin{aligned}
\mathbf{D}^{0}\left(\mathbf{Q}, \omega, z, z^{\prime}\right)= & -\frac{4 \pi c}{\omega^{2}} \delta\left(z-z^{\prime}\right) \mathbf{z} \cdot \mathbf{z} \\
& +\frac{2 \pi i}{c \beta}\left\{\mathbf{e}_{s} \cdot \mathbf{e}_{s}+\mathbf{e}_{p} \cdot \mathbf{e}_{p}\right\} e^{i \beta\left|z-z^{\prime}\right|}
\end{aligned}
$$

Here, the unit vectors are adapted to the geometry of the system such that $\mathbf{e}_{s}=\mathbf{Q}_{0} \times \mathbf{z}$ and $\mathbf{e}_{p}=\frac{c}{\omega}\left[-\beta \operatorname{sgn}\left(z-z^{\prime}\right) \mathbf{Q}_{0}+Q \mathbf{z}\right]$ (where $\mathbf{Q}_{0}$ is the unit vector in the $\mathbf{Q}$ direction) represent directions of $\mathbf{s}(\mathrm{TE})$ and $\mathbf{p}(\mathrm{TM})$ polarized fields, respectively.

We see that the $z$ integration in (20) is restricted exactly from $-L / 2$ to $L / 2$ which implies that the current fluctuation created in the region $-L / 2<z_{1}<L / 2$ can interact via photon propagator $\mathbf{D}^{0}\left(\mathbf{Q}, \omega, z_{1}, z_{2}\right)$ (even though the induced electromagnetic field spreads over all space) only with the current fluctuation in the region $L / 2<z_{2}<L / 2$. This restriction guarantees that $\Pi$ contains information only about the electromagnetic modes characteristic for the electronic system limited to the region $-L / 2<z<L / 2$ (e.g., q2D systems). 
The Dyson equation (20) can be additionally Fourier transformed in the $z$ direction, so that it becomes a full matrix equation

$$
\begin{aligned}
\Pi_{\mathbf{G}, \mathbf{G}^{\prime}}(\mathbf{Q}, \omega)= & \Pi_{\mathbf{G}^{\prime}, \mathbf{G}^{\prime}}^{0}(\mathbf{Q}, \omega) \\
& +\sum_{\mathbf{G}_{1}, \mathbf{G}_{2}} \Pi_{\mathbf{G}, \mathbf{G}_{1}}^{0}(\mathbf{Q}, \omega) \mathbf{D}_{\mathbf{G}_{1}, \mathbf{G}_{2}}^{0}(\mathbf{Q}, \omega) \Pi_{\mathbf{G}_{2}, \mathbf{G}^{\prime}}(\mathbf{Q}, \omega),
\end{aligned}
$$

where $\mathbf{G}=\left(\mathbf{G}_{\|}, G_{z}\right)$ are 3D reciprocal lattice vectors. Here,

$$
\Pi_{\mathbf{G}, \mathbf{G}^{\prime}}^{0}(\mathbf{Q}, \omega)=\Pi_{\mathbf{G}, \mathbf{G}^{\prime}}^{\mathrm{para}}(\mathbf{Q}, \omega)+\Pi_{\mathbf{G}, \mathbf{G}^{\prime}}^{\mathrm{dia}}(\mathbf{Q}, \omega)
$$

represents the Fourier transform of the current-current response tensor (9), and where the Fourier transform of photon propagator can be obtained using (21), (22), and

$$
\mathbf{D}_{\mathbf{G}, \mathbf{G}^{\prime}}^{0}(\mathbf{Q}, \omega)=\frac{1}{L} \int_{-L / 2}^{L / 2} d z d z^{\prime} e^{-i G_{z} z} \mathbf{D}_{\mathbf{G}_{\|}, \mathbf{G}_{\|}^{\prime}}^{0}\left(\mathbf{Q}, \omega, z, z^{\prime}\right) e^{i G_{z}^{\prime} z^{\prime}} .
$$

Using (6), (8), and (11), the Fourier transform of the paramagnetic contribution to the current-current response tensor becomes

$$
\begin{aligned}
\Pi_{\mu \nu, \mathbf{G G}^{\prime}}^{\mathrm{para}}(\mathbf{Q}, \omega)= & -\frac{2}{\Omega \mathrm{c}} \sum_{\mathbf{K}, n, m} \frac{f_{n \mathbf{K}}-f_{m \mathbf{K}+\mathbf{Q}}}{\hbar \omega+i \eta+E_{n \mathbf{K}}-E_{m \mathbf{K}+\mathbf{Q}}} \\
& \times j_{n \mathbf{K}, m \mathbf{K}+\mathbf{Q}}^{\mu}(\mathbf{G})\left[j_{n \mathbf{K}, m \mathbf{K}+\mathbf{Q}}^{\nu}\left(\mathbf{G}^{\prime}\right)\right]^{*},
\end{aligned}
$$

where the current vertices are

$$
j_{n \mathbf{K}, m \mathbf{K}+\mathbf{Q}}^{\mu}(\mathbf{G})=\int_{\Omega} d \mathbf{r} e^{-i\left(\mathbf{Q}+\mathbf{G}_{\|}\right) \cdot \boldsymbol{\rho}-i G_{z} z} j_{n \mathbf{K}, m \mathbf{K}+\mathbf{Q}}^{\mu}(\mathbf{r}),
$$

and where

$$
\begin{aligned}
j_{n \mathbf{K}, m \mathbf{K}+\mathbf{Q}}^{\mu}(\mathbf{r})= & \frac{\hbar e}{2 i m}\left\{\phi_{n \mathbf{K}}^{*}(\mathbf{r}) \partial_{\mu} \phi_{m \mathbf{K}+\mathbf{Q}}(\mathbf{r})\right. \\
& \left.-\left[\partial_{\mu} \phi_{n \mathbf{K}}^{*}(\mathbf{r})\right] \phi_{m} \mathbf{K}+\mathbf{Q}(\mathbf{r})\right\} .
\end{aligned}
$$

Here, the $3 \mathrm{D}$ position vector is $\mathbf{r}=(\boldsymbol{\rho}, z)$. Using (7) and (8), the Fourier transform of the diamagnetic contribution to the current-current response tensor becomes

$$
\Pi_{\mu \nu, \mathbf{G G}^{\prime}}^{\mathrm{dia}}(\mathbf{Q})=-\delta_{\mu \nu} \frac{2 e^{2}}{m c \Omega} \sum_{\mathbf{K}, n} f_{n}(\mathbf{K}) \rho_{n \mathbf{K}, n \mathbf{K}}\left(\mathbf{G}-\mathbf{G}^{\prime}\right),
$$

where the charge vertices are

$$
\rho_{n \mathbf{K}, m \mathbf{K}+\mathbf{Q}}(\mathbf{G})=\int_{\Omega} d \mathbf{r} e^{-i\left(\mathbf{Q}+\mathbf{G}_{\|}\right) \cdot \boldsymbol{\rho}-i G_{z} z} \phi_{n \mathbf{K}}^{*}(\mathbf{r}) \phi_{m \mathbf{K}+\mathbf{Q}}(\mathbf{r}) .
$$

Here, $\Omega=S \times L$ is the normalization volume, $S$ is the normalization surface, and $f_{n \mathbf{K}}=\left(e^{\left(E_{n \mathbf{K}}-E_{F}\right) / k T}+1\right)^{-1}$ is the Fermi-Dirac distribution at temperature $T$. Integrations in (27) and (30) are performed over the normalization volume $\Omega$. Plane-wave expansion of the wave function has the form

$$
\Phi_{n \mathbf{K}}(\boldsymbol{\rho}, z)=\frac{1}{\sqrt{\Omega}} e^{i \mathbf{K} \cdot \rho} \sum_{\mathbf{G}} C_{n \mathbf{K}}(\mathbf{G}) e^{i \mathbf{G} \cdot \mathbf{r}},
$$

where the coefficients $C_{n \mathbf{K}}$ are obtained by solving the KS equations self-consistently. For now, we use $\eta$ as a positive infinitesimal parameter $\left(\eta \rightarrow 0^{+}\right)$, while later we will account for the relaxation processes that occur in the real situations by switching to RTA $(\eta>0)$.

\section{Optical absorption spectrum and conductivity tensor}

If the layered system is in interaction with external electromagnetic field described by the vector potential $\mathbf{A}^{\mathrm{ext}}(\mathbf{r}, t)$, the power at which the external electromagnetic energy is absorbed in the system can be obtained from the classical expression

$$
P(t)=\int d \mathbf{r}_{1} \mathbf{E}^{\text {ext }}\left(\mathbf{r}_{1}, t\right) \cdot \mathbf{j}^{\text {ind }}\left(\mathbf{r}_{1}, t\right),
$$

where in the $\Phi=0$ gauge the external electrical field can be calculated from

$$
\mathbf{E}^{\mathrm{ext}}=-\frac{1}{\mathrm{c}} \frac{\partial \mathbf{A}^{\mathrm{ext}}}{\partial t} .
$$

After inserting the induced current (16) into (31) the absorption power becomes [48]

$$
\begin{aligned}
P(t)= & \int_{-\infty}^{\infty} d t_{1} \\
& \times \int d \mathbf{r}_{1} d \mathbf{r}_{2} \mathbf{E}^{\mathrm{ext}}\left(\mathbf{r}_{1}, t\right) \Pi\left(\mathbf{r}_{1}, \mathbf{r}_{2}, t-t_{1}\right) \mathbf{A}^{\mathrm{ext}}\left(\mathbf{r}_{2}, t_{1}\right) .
\end{aligned}
$$

Suppose now that the crystal layer planes lie parallel to the $x y$ plane, as shown in Fig. 3(a), and the incident electromagnetic field is a plane wave of unit amplitude and polarization $\mathbf{e}$ :

$$
\mathbf{E}^{\mathrm{ext}}(\mathbf{r}, t)=\mathbf{e} \cos (\mathbf{q} \cdot \mathbf{r}-\omega t),
$$

where the incident wave vector is $\mathbf{q}=(\mathbf{Q}, \beta)$ and $\mathbf{Q}=$ $\left(Q_{0 x}, Q_{0 y}\right)$ is the wave vector parallel to the $x y$ plane. The dispersion relation for electromagnetic waves in vacuum $\omega=|\mathbf{q}| \mathbf{c}$ leads to the relation $\beta=\sqrt{\frac{\omega^{2}}{c^{2}}-Q^{2}}$ where $\mathbf{Q}=|\mathbf{Q}|$. This implies that for $\omega>$ Qc the perturbing field has radiative character with respect to the $z$ axis, as sketched in Fig. 3(b), and for $\omega<$ Qc it has evanescent character as sketched in Fig. 3(c). The radiative field can excite optically active modes, such as bright excitons, while the evanescent field is suitable for excitation of collective modes such as polaritons. The unit vector e represents the polarization of incident electromagnetic field. After combining Eqs. (32), (33), and (34), we do the Fourier transform of the current-current response tensor $\Pi(\omega)=\int_{-\infty}^{\infty} d t e^{i \omega\left(t-t^{\prime}\right)} \Pi\left(t-t^{\prime}\right)$ and the expression for the absorption power becomes

$$
\begin{aligned}
P(\omega)= & \frac{c}{2 \omega} \operatorname{Im}\left\{\sum_{\mu, \nu} e_{\mu} e_{\nu}\right. \\
& \left.\times \int d \mathbf{r}_{1} \mathbf{r}_{2} e^{-i \mathbf{q} \cdot \mathbf{r}_{1}} \Pi_{\mu \nu}\left(\mathbf{r}_{1}, \mathbf{r}_{2}, \omega\right) e^{i \mathbf{q} \cdot \mathbf{r}_{2}}\right\} .
\end{aligned}
$$

Fourier transforming Eqs. (16) and (32) in $\omega$ space and combining them we obtain

$$
j_{\mu}^{\text {ind }}(\mathbf{r}, \omega)=-i \frac{c}{\omega} \sum_{\nu} \int d \mathbf{r}_{1} \Pi_{\mu \nu}\left(\mathbf{r}, \mathbf{r}_{1}, \omega\right) E_{\nu}^{\mathrm{ext}}\left(\mathbf{r}_{1}, \omega\right) .
$$


Using the fact that the induced current can also be calculated in terms of electrical conductivity tensor as

$$
\mathbf{j}_{\mu}^{\text {ind }}=\sum_{v} \sigma_{\mu \nu} \otimes E_{v}^{\mathrm{ext}},
$$

we obtain the useful connection between the conductivity tensor and current-current response tensor

$$
\sigma_{\mu \nu}\left(\mathbf{r}, \mathbf{r}^{\prime}, \omega\right)=-i \frac{c}{\omega} \Pi_{\mu \nu}\left(\mathbf{r}, \mathbf{r}^{\prime}, \omega\right)
$$

Now, we want to exploit our results for the tensor $\Pi_{\mu \nu, \mathbf{G G}^{\prime}}(\mathbf{Q}, \omega)$. Fourier transforming it to the real space:

$$
\begin{aligned}
\Pi_{\mu \nu}\left(\mathbf{r}, \mathbf{r}^{\prime}, \omega\right)= & \frac{1}{L} \sum_{\mathbf{G}, \mathbf{G}^{\prime}} \int \frac{d \mathbf{Q}}{(2 \pi)^{2}} e^{i(\mathbf{Q}+\mathbf{G}) \cdot \mathbf{r}} \\
& \times e^{-i\left(\mathbf{Q}+\mathbf{G}^{\prime}\right) \cdot \mathbf{r}^{\prime}} \Pi_{\mu \nu, \mathbf{G} \mathbf{G}^{\prime}}(\mathbf{Q}, \omega)
\end{aligned}
$$

and inserting in (35) the absorption power per unit area becomes

$$
P(\mathbf{Q}, \omega)=\frac{c}{2 \omega L} S(\mathbf{Q}, \omega),
$$

where the spectral function is

$S(\mathbf{Q}, \omega)=\operatorname{Im}\left\{\sum_{\mu, \nu} e_{\mu} e_{\nu} \sum_{G_{z}, G_{z}^{\prime}} F\left(G_{z}\right) F\left(G_{z}^{\prime}\right) \Pi_{\mu \nu, G_{z} G_{z}^{\prime}}(\mathbf{Q}, \omega)\right\}$

and the form factors are $F\left(G_{z}\right)=\frac{2}{G_{z}+\beta} \sin \frac{\left(G_{z}+\beta\right) L}{2}$. It is more convenient to deal with absorption coefficient $A(\mathbf{Q}, \omega)=$ $P(\mathbf{Q}, \omega) /|\mathcal{P}|$ where the incident flux of electromagnetic energy (Poynting vector) is given by $\mathcal{P}=\frac{c}{4 \pi} \mathbf{E} \times \mathbf{B}$. For unit amplitude incident electrical field (34) the incident flux is $|\mathcal{P}|=\frac{c}{8 \pi}$ and the absorption coefficient is

$$
A(\mathbf{Q}, \omega)=\frac{4 \pi}{\omega L} S(\mathbf{Q}, \omega) .
$$

The Fourier transform of the conductivity tensor (38) can be obtained directly from the Fourier transform of current-current response tensor as

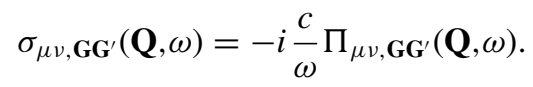

The current which is induced by a homogeneous electric field $\mathbf{E}=\mathbf{e} \cos \omega t$ [which corresponds to electromagnetic field (34)] which is incident normally $(\mathbf{Q}=0)$ and for $\beta \ll 1 / L$ can be, using (37) and (43), written as

$$
j_{\mu}^{\text {ind }}(z, \omega)=\frac{c}{\omega} \sum_{G_{z}} e^{i G_{z} z} \sum_{\nu} \operatorname{Im} \Pi_{\mu \nu, G_{z} 0}(\mathbf{Q}=0, \omega) e_{\nu} .
$$

Here, as we are not interested in the current variation within the unit cell in the parallel $x y$ direction, we retained only $\mathbf{G}_{\|}=\mathbf{G}_{\|}^{\prime}=0$ components. If the field is directed along the $x$ or $y$ axis, the current flow per unit thickness of the sample can be obtained by $z$ integration in (44), when it becomes

$$
\operatorname{Re} \sigma_{\mu \mu}(\omega)=\frac{c L}{\omega} \operatorname{Im}_{\mu \mu, 00}(\mathbf{Q}=0, \omega), \quad \mu=x, y
$$

which corresponds to the experimentally measurable conductivity.

\section{Alternative expression for the current-current response tensor}

For numerical reasons, a straightforward calculation of the current-current response tensor (24) from Eqs. (26)-(30) can lead to nonphysical results, so here we shall derive an alternative expression which avoids this problem. Namely, the expression for $\Pi^{\text {para }}$ (26) includes summation over all unoccupied bands, which is not the case for $\Pi^{\text {dia }}$ (29), so if the calculation is not performed with the same high precision the result for $\Pi$ (24) might be erroneous.

Suppose for the moment that the system interacts only with the external electromagnetic field $\mathbf{A}^{\text {ext }}(\mathbf{r}, t)$ and that the interaction $V^{\text {in }}$ is neglected. Then, the induced current and charge distributions are given by (16) and (17), respectively. After inserting (16) and (17) into the continuity equation (19) and performing the Fourier transform in $(\mathbf{q}, \omega)$ space, it becomes [45]

$$
\omega \Pi_{0 \nu, \mathbf{G G}^{\prime}}^{0}(\mathbf{Q}, \omega)=\sum_{\mu} q_{\mu} \Pi_{\mu \nu, \mathbf{G G}^{\prime}}^{0}(\mathbf{Q}, \omega),
$$

where $\left(q_{x}, q_{y}, q_{z}\right)=\left(\mathbf{Q}+\mathbf{G}_{\|}, G_{z}\right)$ and $v=x, y, z$. The Fourier transform of the current-current response tensor $\Pi_{\mu \nu}$ is given by (24), (26), and (29) and the Fourier transform of the chargecurrent response tensor $\Pi_{0 v}$, defined by (18), is given by

$$
\begin{aligned}
& \Pi_{0 v, \mathbf{G G}^{\prime}}^{0}(\mathbf{Q}, \omega)=-\frac{2}{\Omega \mathrm{c}} \sum_{\mathbf{K}, n, m} \frac{f_{n \mathbf{K}}-f_{m \mathbf{K}+\mathbf{Q}}}{\hbar \omega+i \eta+E_{n \mathbf{K}}-E_{m \mathbf{K}+\mathbf{Q}}} \\
& \times \rho_{n \mathbf{K}, m \mathbf{K}+\mathbf{Q}}(\mathbf{G})\left[j_{n \mathbf{K}, m \mathbf{K}+\mathbf{Q}}^{\nu}\left(\mathbf{G}^{\prime}\right)\right]^{*},
\end{aligned}
$$

where the current and charge vertices are defined by (27) and (30), respectively. In the static limit $(\omega=0)$, when the current flow becomes stationary (the dc limit) the continuity equation (46) becomes

$$
\sum_{\mu} q_{\mu} \Pi_{\mu \nu, \mathbf{G G}^{\prime}}^{0}(\mathbf{Q}, 0)=0 .
$$

It is important to note that $\Pi_{\mu \nu}^{0}$ should be calculated so that condition (48) is satisfied very accurately, otherwise it can lead to some incorrect physical conclusions. For example, using the definition of the conductivity tensor (38), the dc conductivity becomes

$$
\sigma_{\mu \nu, \mathbf{G G}^{\prime}}(\mathbf{Q}, \omega \rightarrow 0)=-i c \lim _{\omega \rightarrow 0} \frac{\Pi_{\mu \nu, \mathbf{G G}^{\prime}}(\mathbf{Q}, \omega)}{\omega} .
$$

We see that if the condition (48) is not satisfied, (49) could lead to the wrong conclusion about, e.g., the existence of superconducting state $(\sigma \rightarrow \infty)$. Also, in the normal-metal state it could affect the Drude plasmon frequency. As already mentioned, the problem arises from the numerical calculation of the paramagnetic term (26) which includes summation over all unoccupied bands and will never be capable to cancel exactly the diamagnetic contribution (29) which includes only the summation over occupied states and can be calculated very accurately.

This problem can be solved so that we calculate the paramagnetic term at some appropriate level of accuracy and then require the diamagnetic term to satisfy the continuity equation (48), i.e.,

$$
\Pi_{\mu \nu, \mathbf{G G}^{\prime}}^{\mathrm{dia}}(\mathbf{Q})=-\Pi_{\mu \nu, \mathbf{G G}^{\prime}}^{\mathrm{para}}(\mathbf{Q}, 0) .
$$


After using (24), (26), and (50), the redefined current-current response tensor becomes

$$
\begin{aligned}
\Pi_{\mu \nu, \mathbf{G G}^{\prime}}^{0}(\mathbf{Q}, \omega)= & \frac{2}{\Omega \mathrm{c}} \sum_{\mathbf{K}, n, m} \frac{\hbar \omega}{E_{n \mathbf{K}}-E_{m \mathbf{K}+\mathbf{Q}}} \\
& \times \frac{f_{n \mathbf{K}}-f_{m \mathbf{K}+\mathbf{Q}}}{\hbar \omega+i \eta+E_{n \mathbf{K}}-E_{m} \mathbf{K}+\mathbf{Q}} \\
& \times j_{n \mathbf{K}, m \mathbf{K}+\mathbf{Q}}^{\mu}(\mathbf{G})\left[j_{n \mathbf{K}, m \mathbf{K}+\mathbf{Q}^{\nu}}\left(\mathbf{G}^{\prime}\right)\right]^{*} .
\end{aligned}
$$

Now, we shall demonstrate that the current-current response tensor given by (51) satisfies the continuity equation in the whole frequency range, i.e., it satisfies Eq. (46). We start from the operator form of the continuity equation, which for the system of independent electrons and without interaction with the external field can be written as

$$
\left[\rho(\mathbf{r}, t), H_{0}^{e}\right]=\frac{i}{\hbar} \nabla \mathbf{j}^{\text {para }}(\mathbf{r}, t),
$$

where $\hat{O}(t)=\{\rho(t), \mathbf{j}(t)\}$ are Heisenberg operators, defined as

$$
\hat{O}(t)=e^{i H_{0}^{e} t} \hat{A} e^{i H_{0}^{e} t} .
$$

The Schrödinger operators $\hat{O}=\{\rho, \mathbf{j}\}$ are defined as (6) and (7) and the Hamiltonian $H_{0}^{e}$ is given by (2). After Fourier transformation of Eq. (52), i.e., $\int d \mathbf{r} e^{-i \mathbf{q r}}\left\{\left[\rho(\mathbf{r}, t), H_{0}^{e}\right]=\frac{i}{\hbar} \nabla \mathbf{j}(\mathbf{r}, t)\right\}$, and some commutation relations manipulation it becomes

$$
\begin{array}{r}
\sum_{\mathbf{K}, n, m} \rho_{n \mathbf{K}, m \mathbf{K}+\mathbf{Q}}(\mathbf{G})\left[E_{n \mathbf{K}}-E_{m \mathbf{K}+\mathbf{Q}}\right] c_{n \mathbf{K}}^{\dagger} c_{m \mathbf{K}+\mathbf{Q}} \\
=-\sum_{\mathbf{K}, n, m} \sum_{\mu} \hbar q_{\mu} j_{n \mathbf{K}, m \mathbf{K}+\mathbf{Q}}^{\mu}(\mathbf{G}) c_{n \mathbf{K}}^{\dagger} c_{m \mathbf{K}+\mathbf{Q} .}
\end{array}
$$

For clarity, we omit to write time variables in the Heisenberg creation and annihilation operators which, after using definition (53), are $c_{n \mathbf{K}}^{\dagger}(t)=c_{n \mathbf{K}}^{\dagger} e^{i \frac{E_{n \mathbf{K}}}{\hbar} t}$ and $c_{n \mathbf{K}}(t)=c_{n \mathbf{K}} e^{-i \frac{E_{n \mathbf{K}}}{\hbar} t}$ where $c_{n \mathbf{K}}^{\dagger}$ and $c_{n \mathbf{K}}$ are Schrödinger operators.

By equating left and right sides in (54) we obtain a useful connection between the charge and current vertices

$$
\rho_{n \mathbf{K}, m \mathbf{K}+\mathbf{Q}}(\mathbf{G})=-\sum_{\mu} \hbar q_{\mu} \frac{j_{n \mathbf{K}, m \mathbf{K}+\mathbf{Q}}^{\mu}(\mathbf{G})}{E_{n \mathbf{K}}-E_{m \mathbf{K}+\mathbf{Q}}} .
$$

After inserting charge vertices (55) into charge-current response function (47) and then into the left-hand side of the continuity equation (46) it becomes exactly equal to its right-hand side in which the current-current response tensor (51) is inserted. Therefore, current-current response tensor (51) satisfies the continuity equation for any $\omega$.

An important aspect of the new current-current response tensor (51) is the appearance of the $\hbar \omega /\left(E_{n \mathbf{K}}-E_{m \mathbf{K}+\mathbf{Q}}\right)$ prefactor which ensures that $\Pi_{\mu \nu}^{0}(\mathbf{Q}, \omega) \rightarrow 0$ when $\omega \rightarrow 0$ and also naturally compensates the $\omega^{-1}$ divergence in the Kubo conductivity formula.

\section{E. Long-wavelength limit $\mathbf{Q} \approx 0$}

We shall now analyze the long-wavelength limit $\mathbf{Q} \approx 0$ of the redefined current-current response tensor (51). First, it is convenient to decompose the current-current response tensor into its intraband $(n=m)$ and interband $(n \neq m)$ contributions

$$
\Pi_{\mu \nu, \mathbf{G G}^{\prime}}^{0}(\mathbf{Q}, \omega)=\Pi_{\mu \nu, \mathbf{G G}^{\prime}}^{0, \text { intra }}(\mathbf{Q}, \omega)+\Pi_{\mu \nu, \mathbf{G G}^{\prime}}^{0, \text { inter }}(\mathbf{Q}, \omega) .
$$

For $\mathbf{Q} \approx 0$ and $n=m$, we have that $E_{n \mathbf{K}}-E_{m \mathbf{K}+\mathbf{Q}} \approx 0$, and we can write the intraband contribution in RTA as $[35,36]$

$$
\begin{aligned}
\Pi_{\mu \nu, \mathbf{G G}^{\prime}}^{0, \text { intra }}(\omega)= & \frac{2}{\Omega \mathrm{c}} \frac{\hbar \omega}{\hbar \omega+i \eta_{\text {intra }}} \sum_{\mathbf{K}, n} \frac{\partial f\left(E_{n \mathbf{K}}\right)}{\partial E_{n \mathbf{K}}} \\
& \times j_{n \mathbf{K}, n \mathbf{K}}^{\mu}(\mathbf{G})\left[j_{n \mathbf{K}, n \mathbf{K}}^{v}\left(\mathbf{G}^{\prime}\right)\right]^{*},
\end{aligned}
$$

where we changed $f_{n \mathbf{K}} \rightarrow f\left(E_{n \mathbf{K}}\right)$, and for simplicity we write $\Pi_{\mu \nu, \mathbf{G G}^{\prime}}^{0}(\mathbf{Q} \approx 0, \omega) \equiv \Pi_{\mu \nu, \mathbf{G G}^{\prime}}^{0}(\omega)$. This intraband term leads to the Drude conductivity formula, as will be shown following. For $n \neq m$ we have

$$
\begin{aligned}
\Pi_{\mu \nu, \mathbf{G G}^{\prime}}^{0, \text { inter }}= & \frac{2}{\Omega \mathrm{c}} \sum_{\mathbf{K}, n \neq m} \frac{\hbar \omega}{E_{n \mathbf{K}}-E_{m \mathbf{K}}} \\
& \times \frac{f_{n \mathbf{K}}-f_{m \mathbf{K}}}{\hbar \omega+i \eta_{\text {inter }}+E_{n \mathbf{K}}-E_{m \mathbf{K}}} \\
& \times j_{n \mathbf{K}, m \mathbf{K}}^{\mu}(\mathbf{G})\left[j_{n \mathbf{K}, m \mathbf{K}}^{\nu}\left(\mathbf{G}^{\prime}\right)\right]^{*} .
\end{aligned}
$$

The above expression has a proper behavior for $\omega<\mid E_{n \mathbf{K}}-$ $E_{m \mathbf{K}} \mid$, but only in the ideal (undamped) regime, i.e.. $\eta_{\text {inter }} \rightarrow$ $0^{+}$, or if $\eta_{\text {inter }}$ is much smaller than the interband threshold energy. If this condition is not met, the real part of interband conductivity $\operatorname{Re} \sigma^{\text {inter }}(\omega)$ derived from Eq. (58) will give spurious estimation in the $\omega \approx 0$ regime. To get a proper behavior of Eq. (58) in this regime, one would need to go beyond RTA and include the vertex corrections and single-particle self-energies for the relevant scattering processes. In the case of doped graphene, the expression (58) can be safely used only if $\eta_{\text {inter }}$ is sufficiently smaller than the Fermi energy, i.e., $\eta_{\text {inter }}<E_{F}$ [35]. For pristine graphene, where the interband threshold energy goes to 0 , it is safer to use the usual interband contribution to the current-current correlation function [49-52]

$$
\begin{aligned}
\Pi_{\mu \nu, \mathbf{G G}^{\prime}}^{0, \text { inter }}(\omega)= & \frac{2}{\Omega \mathrm{c}} \sum_{\mathbf{K}, n \neq m} \frac{(\hbar \omega)^{2}}{E_{n \mathbf{K}}-E_{m \mathbf{K}}} \\
& \times \frac{f_{n \mathbf{K}}-f_{m \mathbf{K}}}{\left(\hbar \omega+i \eta_{\text {inter })^{2}}-\left(E_{n \mathbf{K}}-E_{m \mathbf{K}}\right)^{2}\right.} \\
& \times j_{n \mathbf{K}, m \mathbf{K}}^{\mu}(\mathbf{G})\left[j_{n \mathbf{K}, m \mathbf{K}}^{v}\left(\mathbf{G}^{\prime}\right)\right]^{*} .
\end{aligned}
$$

One can easily see that the above expression for the interband term has a more appropriate behavior in the static limit $(\omega=0)$ than expression (58) [53]. The expressions (57) and (59) will be used to calculate the adsorption coefficient (42) and conductivity (45) for optical wave vectors $\mathbf{Q} \sim \mathbf{Q}_{\text {light }}$. Additionally, we will use Eq. (58) for comparison.

In addition, we shall verify that (51) leads to the correct dielectric function and conductivity in the three-dimensional electron gas in the long-wavelength $(\mathbf{q} \approx 0)$ limit. For a polarizable system of arbitrary symmetry (in the linear-response approximation), the electric field $\mathbf{E}$ and electric displacement $\mathcal{D}$ can be related as

$$
\mathcal{D}=\boldsymbol{\varepsilon} \otimes \mathbf{E},
$$

where $\varepsilon$ is the nonlocal dielectric tensor and $\otimes$ is matrix multiplication and convolution in real space. After combining 
the definition (60) with the Maxwell and Dyson equations for electrical field $\mathbf{E}$ in the presence of a polarizable system, we can obtain a general relationship between the dielectric tensor and the current-current response tensor [14,54]

$$
\varepsilon_{\mu \nu}\left(\mathbf{r}, \mathbf{r}^{\prime}, \omega\right)=\delta\left(\mathbf{r}-\mathbf{r}^{\prime}\right) \delta_{\mu \nu}+\frac{4 \pi c}{\omega^{2}} \Pi_{\mu \nu}^{0}\left(\mathbf{r}, \mathbf{r}^{\prime}, \omega\right) .
$$

If we consider a 3D homogeneous electron gas the Fourier transform of (61) can be written as

$$
\varepsilon_{\mu \nu}(\mathbf{q}, \omega)=\delta_{\mu \nu}+\frac{4 \pi c}{\omega^{2}} \Pi_{\mu \nu}^{0}(\mathbf{q}, \omega),
$$

where $\Pi_{\mu \nu}^{0}(\mathbf{q}, \omega)$ can be obtained from (51), $\mathbf{G}=\mathbf{G}^{\prime}=0$, $\mathbf{K} \rightarrow \mathbf{k}$ becomes a $3 \mathrm{D}$ wave vector, and $\mathbf{Q} \rightarrow \mathbf{q}$ becomes 3D momentum transfer. Also, after using the fact that in a homogeneous electron gas there is only one $(n=m=1)$ parabolic band $\left(E_{\mathbf{k}}=\frac{\hbar^{2} \mathbf{k}^{2}}{2 m}\right)$ and the wave functions are plane waves $\left[\Phi_{\mathbf{k}}(\mathbf{r})=\frac{1}{\sqrt{\Omega}} e^{i \mathbf{k r}}\right]$, the current-current response tensor (51) becomes

$$
\begin{aligned}
\Pi_{\mu \nu}^{0}(\mathbf{q}, \omega)= & \frac{\hbar^{3} \omega e^{2}}{2 m^{2} \Omega \mathrm{c}} \sum_{\mathbf{k}} \frac{\left(2 k_{\mu}+q_{\mu}\right)\left(2 k_{v}+q_{\nu}\right)}{E_{\mathbf{k}}-E_{\mathbf{k}+\mathbf{q}}} \\
& \times \frac{f_{\mathbf{k}}-f_{\mathbf{k}+\mathbf{q}}}{\hbar \omega+i \eta+E_{\mathbf{k}}-E_{\mathbf{k}+\mathbf{q}}},
\end{aligned}
$$

where we also used the definition of the current vertices (27) and (28). One can easily show that such a current-current response tensor in the long-wavelength limit becomes

$$
\Pi_{\mu \nu}^{0}(\mathbf{q} \approx 0, \omega)=-\frac{n e^{2}}{m c} \frac{\omega}{\omega+i \eta} \delta_{\mu \nu},
$$

where $n=\frac{1}{\Omega} \sum_{\mathbf{k}} f(\mathbf{k})$ is the electron density. After inserting (64) into (62), we get the well-known Drude dielectric function

$$
\varepsilon_{\mu \nu}(\mathbf{q} \approx 0, \omega)=\left[1-\frac{\omega_{p}^{2}}{\omega(\omega+i \eta)}\right] \delta_{\mu \nu},
$$

where $\omega_{p}=\sqrt{4 \pi n e^{2} / m}$ is the bulk plasmon frequency. Similarly, combining (64) and the definition (38) leads to the Drude conductivity tensor [55]

$$
\sigma_{\mu \nu}(\mathbf{q} \approx 0, \omega)=i \frac{n e^{2}}{m} \frac{1}{\omega+i \eta} \delta_{\mu \nu} .
$$

From the definition of the simple Drude dc conductivity $\sigma=$ $\frac{n e^{2}}{m} \tau$ it is obvious that $\eta$ plays the role of the inverse relaxation time, i.e., $\eta=1 / \tau$.

\section{RESULTS AND DISCUSSION}

In order to test our theoretical approach and demonstrate some of its advantages, we shall apply it to calculate optical properties of a freestanding single-layer graphene.

\section{A. Computational details}

The first part of the calculation consists of determining the KS ground state of the single-layer graphene and the corresponding wave functions and energies. For the unit-cell constant we use the experimental value of $a=4.651$ a.u. [56], and we separate the graphene layers with the distance
$L=5 a$. For calculating KS wave functions and energies, we use a plane-wave self-consistent field DFT code (PWSCF) within the QUANTUM ESPRESSO (QE) package [57]. The coreelectron interaction was approximated by the norm-conserving pseudopotentials [58], and the exchange correlation (XC) potential by the Perdew-Zunger local density approximation (LDA) [59]. To calculate the ground-state electronic density we use $30 \times 30 \times 1$ Monkhorst-Pack $K$-point mesh [60] of the first Brillouin zone (BZ), and for the plane-wave cutoff energy we choose $50 \mathrm{Ry}$. In order to achieve better resolution in the low energy and the static limit $(\omega \rightarrow 0)$, the current response tensor (51) is evaluated from the wave functions $\phi_{n \mathbf{K}}(\mathbf{r})$ and energies $E_{n}(\mathbf{K})$ calculated for the $402 \times 402 \times 1$ MonkhorstPack $K$-point mesh, and band summation is performed over 30 bands. In the calculation, we use two kinds of damping rates which account for the relaxation processes in our system (RTA): $\eta_{\text {intra }}$ for transitions within the same band $(n \rightarrow n)$, and $\eta_{\text {inter }}$ for transitions between different bands $(n \rightarrow m)$. In principle, if the effects of single-particle self-energies and vertex corrections, coming from electron scattering on impurities, other electrons, or phonons, are included in the current-current correlation function, the two damping rates can be expressed as the imaginary parts of the difference between the electron (retarded, $R$ ) and hole (advanced, $A$ ) self-energies $\eta_{n m}(\mathbf{K}, \mathbf{Q}, \omega) \approx \operatorname{Im} \Sigma_{n \mathbf{K}}^{R}(\omega)-\operatorname{Im} \Sigma_{m \mathbf{K}+\mathbf{Q}}^{A}(\omega)$. These electronhole self-energies are closely related to the memory function mentioned in the Introduction of this paper [36]. In our case, they will be variable parameters, chosen to fit the experimental data. During this and our previous investigations [30,31] we noticed that in the optical or long-wavelength limit $(\mathrm{Q} \approx 0)$, the crystal local field effects in the parallel direction $(x y$ plane) are negligible in almost whole frequency range $\omega$. It is so because in the $\mathrm{Q} \approx 0$ limit the induced electrical field variation on the scale of unit-cell size $a$ becomes a very smooth function. However, for large wave vectors $Q \sim 1 / a$ the induced field substantially varies within the unit cell, i.e., crystal local field effects become important and should be included in the calculation, as systematically explored in Refs. [61,62]. In Ref. [62], the authors nicely demonstrated how inclusion of the crystal local field effects can substantially influence the plasmon dispersion relation along the Brillouin zone boundary ( $M K$ direction). But here we mostly investigate the optical modes and conductivity, where $\mathrm{Q}=0$ and we can take $G_{\|}=G_{\|}^{\prime}=0$. However, broken symmetry in the $z$ direction results in big inhomogeneity of induced currents and fields in that direction. This requires inclusion of the crystal local field effects in the $z$ direction which we describe with $21 G_{z}$ Fourier components.

After solving the Dyson equation (23), we obtain the screened current-current response tensor $\Pi$ which enters in the absorption coefficient (42). The conductivity tensor (43) for pristine graphene can be calculated from the unscreened current-current response tensor (51) [55].

\section{B. Optical absorption}

In this section, we study the absorption of incident electromagnetic field (34) in the freestanding single-layer graphene. We fix the parallel component of the incident wave vector $\mathbf{Q}=\left(\mathrm{Q}_{0 x}, \mathrm{Q}_{0 y}\right)$ and change the incident frequency $\omega$. 
Due to the relation $\omega=|\mathbf{q}| c$, for $\omega<\mathrm{Qc}$ the perpendicular component of incident wave vector $\beta$ is imaginary, and the incident field has evanescent character (in the $z$ direction), as sketched in Fig. 3(b). For $\omega \geqslant$ Qc, $\beta$ is real and the incident field has radiative character in all three directions [Fig. 3(c)]. In the latter case, the incident wave vector $\mathbf{q}$ is inclined relative to the graphene surface by an angle $\theta$ given by

$$
\sin \theta=\frac{\beta}{|\mathbf{q}|}=\sqrt{1-\frac{\mathrm{Q}^{2} \mathrm{c}^{2}}{\omega^{2}}},
$$

as sketched in Fig. 3(a).

Let us discuss some specific cases. For example, for $\omega=$ Qc (on the light cone) the incident electromagnetic field is a plane wave which propagates parallel to the $x y$ plane $(\theta=0), \mathbf{s}$ polarization is in the $x y$ plane, and $\mathbf{p}$ polarization is wholly in the perpendicular $z$ direction. Also, if $\mathrm{Q}=0$, the electromagnetic field has radiative character in the whole frequency range $(\omega>0)$, incidence is normal to the graphene surface $(\theta=\pi / 2)$, and $\mathbf{s}$ and $\mathbf{p}$ polarizations become equal.

Retardation effects are most pronounced for very small wave vectors, so we shall divide our discussion of optical absorption into two parts: for small $\mathrm{Q} \approx \mathrm{Q}_{\text {light }}$, and large $\mathrm{Q} \gg \mathrm{Q}_{\text {light }}$, wave vectors.

\section{1. $\mathrm{Q} \approx \mathrm{Q}_{\text {light }}$}

Figure 4(a) shows the optical absorption coefficient (42) of $\mathrm{s}(x)$ or $\mathrm{p}(y)$ polarized electromagnetic fields in pristine graphene for normal incidence $\mathrm{Q}=0$. Optical absorption onset appears already at $\omega=0$ which is due to the gapless dipole active $\pi \rightarrow \pi^{*}$ interband transitions near the $K$ point of the BZ. In the IR and visible regions $(\omega<3 \mathrm{eV})$ absorption monotonically increases. The first absorption maximum,

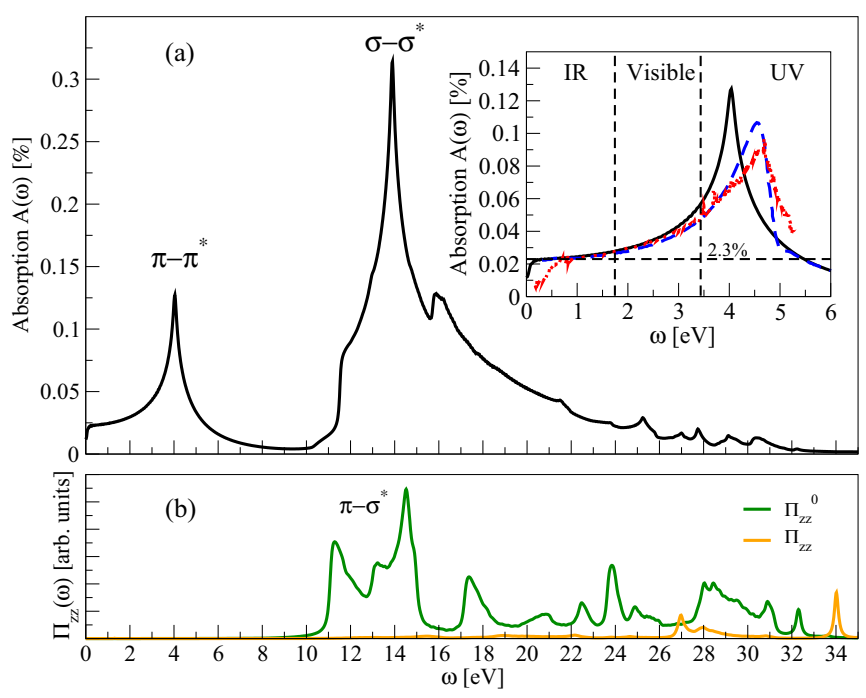

FIG. 4. (a) Absorption spectrum in pristine graphene $\left(E_{F}=0\right)$ for normal incidence $(\mathrm{Q}=0)$ and $T=0 \mathrm{~K}$. Inset: details of optical absorption in IR, visible, and UV regions; black solid lines: this work; blue dashed lines: theoretical results taken from Ref. [15]; and red dotted lines: experimental results taken from Ref. [16]. The damping parameters are $\eta_{\text {intra }}=10 \mathrm{meV}$ and $\eta_{\text {inter }}=50 \mathrm{meV}$. (b) Noninteracting current-current correlation tensor (51) (green line) and the interacting one (23) (yellow line) for $\mu=v=z$ and $\mathrm{Q}=0$. which appears in the ultraviolet (UV) region at $\omega=4 \mathrm{eV}$, is a consequence of transitions between $\pi$ and $\pi^{*}$ bands along the $M M^{\prime}$ and $M \Gamma$ directions of $\mathrm{Q}$, as discussed in detail in [31]. This resonance absorbs about $12 \%$ of incident electromagnetic energy. In the far UV region $\omega>6 \mathrm{eV}$ the spectrum shows more structures, which are due to optically active transitions between $\sigma$ and $\sigma^{*}$ bands, with the main peak at $\omega=13.9 \mathrm{eV}$. This very strong excitation mode absorbs $30 \%$ of the incident electromagnetic energy.

Black solid line in the inset of Fig. 4(a) shows the details of optical absorption in IR, visible, and UV regions. In the whole IR region, the absorption is close to the universal value of $\pi \alpha=$ $2.293 \%$ (where $\alpha=\frac{1}{137}$ is the fine-structure constant), denoted by the horizontal dashed line, as predicted experimentally in Refs. [17,18]. In the far IR region $(\omega<200 \mathrm{meV})$, the absorption begins to decrease faster until it reaches $A(\omega=0)$ value which is about half of the universal value $\pi \alpha$. However, the $A(\omega=0)$ value depends on the damping constants $\eta_{\text {intra }}$ and $\eta_{\text {inter }}$ used in the calculation, which will be shown later in the case of the conductivity formula. This dependence can already be anticipated from the expressions (57), (58), and (59). The blue dashed line is the theoretical result taken from Ref. [15], and the red dotted line is the experimental result taken from Ref. [16]. We see that our absorption maximum is at a substantially lower energy $(4.05 \mathrm{eV})$ than the 4.62-eV peak which appears in both theoretical and experimental spectra. This is because the authors in Ref. [15] in their GW-BSE calculation included quasiparticle corrections of the DFT band structure and also the excitonic effects, i.e., electron-hole interaction. Here, the spectrum is calculated within the RPA which includes screening effects, although this screening is small for pristine graphene in the optical limit $(\mathrm{Q} \approx 0)$ due to reduced dimensionality in comparison with the bulk system like graphite, and also due to reduced number of charge carriers in comparison with doped graphene [e.g., the dielectric function is in this case $\varepsilon(\mathbf{Q} \approx 0, \omega) \approx 1$ ] [31]. We see quite a nice agreement of our results with the theoretical result from Ref. [15] in the whole IR and visible region, which will be the region of our main future interest. However, for $\omega<0.5 \mathrm{eV}$, both theoretical results start disagreeing with the experimental spectrum which suddenly decreases below the universal value. This is probably due to the weak doping which causes a shift of optical absorption onset from $\omega=0$ to $2 E_{F}$. Figure 5 shows our theoretical results obtained using Eqs. (58) (yellow line) and (59) (blue line) for the interband channel, which are then compared with the measured absorption spectrum for a graphene sample (red solid lines) taken from Ref. [18]. We see very good agreement in the frequency region $0.3 \mathrm{eV}<\omega<1.2 \mathrm{eV}$ [especially when using Eq. (59)], however, we have slightly doped our graphene $\left(E_{F}=0.1 \mathrm{eV}\right)$ in order to achieve this result.

We want to emphasize here that the $\Pi_{z z}(\mathbf{Q} \approx 0, \omega)$ component of the full current-current response tensor is negligible in graphene [25], as seen in Fig. 4(b), so it is sufficient to use only $x$ and $y$ components for investigating electromagnetic response in graphene.

Now, we show the results for the two electromagnetic modes in doped graphene, appearing within the window constrained by the intraband and interband continua: the usual TM mode (2D plasmon polariton or 2DPP), shown for 


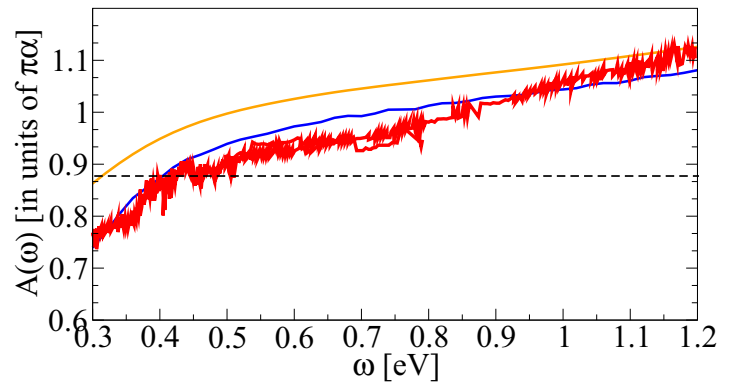

FIG. 5. Calculated absorption spectrum in slightly doped graphene $\left(E_{F}=0.1 \mathrm{eV}\right)$ for normal incidence $(\mathrm{Q}=0)$ and $T=$ $300 \mathrm{~K}$ obtained with Eqs. (58) (yellow line) and (59) (blue line). Experimental absorption spectrum for graphene sample taken from Ref. [18] is represented with red line. Horizontal dashed line denotes the universal absorption constant $\pi \alpha$. The interband damping parameter used in this calculations is $\eta_{\text {inter }}=50 \mathrm{meV}$.

$\mathrm{Q} \sim 10^{-7}$ and $\omega \approx 0$ in Figs. 6(a) and 6(b), and the TE mode $[9,37,38]$, shown for $\mathrm{Q} \sim 10^{-4}$ and $\omega \sim E_{F}$ in Figs. 7(a) and 7(b). It can be seen that the 2DPP mode can be observed only if the incident EM wave is p polarized, while for the same $(Q, \omega)$ values and $\mathbf{s}$ polarized EM wave the only feature appearing is the Drude peak intersected with the light line $(\mathrm{Qc})$. In the insets of Figs. 6(a) and 6(b), we compare the dispersion of the 2DPP with the $\sqrt{\mathrm{Q}} 2 \mathrm{D}$ plasmon dispersion (when $\mathrm{c} \rightarrow \infty$ ). Here, we see how coupling to the light waves influences the longitudinal plasmon mode and how its dispersion changes from $\sqrt{\mathrm{Q}}$ to Qc for very small $\mathrm{Q}$ and $\omega$ when the electronic excitations of graphene are coupled to EM waves [64]. The effects of this coupling can also be seen in the second $\mathbf{s}(\mathrm{TE})$ mode for $\omega \sim E_{F}$. From Figs. 7(a) and 7(b), we see that the energy peak of this mode is always just below Qc, making it almost undistinguishable from the light line. Insets of these figures emphasize this even more. In Ref. [39], the authors have systematically investigated the TE plasmon dispersion relation which exhibits an anisotropic deviation from linearity when the graphene sample is under uniaxial strain [63].

In addition, we present absorption intensities for $\omega \approx 4 \mathrm{eV}$ and $\mathbf{s}$ polarized EM wave, where the peak due to $\pi \rightarrow \pi^{*}$ transitions appears [Fig. 7(c)]. It is clear from the figure that there is no coupling between these transitions and EM waves, which is an additional proof in the pristine graphene plasmon debate, that there is no so-called $\pi$ plasmon for $\mathrm{Q} \approx 0$ while for the large $Q$ wave vectors the plasmon is formed [31,41].

\section{2. $\mathrm{Q} \gg \mathbf{Q}_{\text {light }}$}

Figures 8(a)-8(f) show absorption spectra for different dopings, incident polarizations, and for five different wave vectors along the $\Gamma M$ direction of the first Brillouin zone:

$$
\mathbf{Q}_{n}=n \Delta \mathbf{Q} \hat{\mathbf{y}}, \quad n=0,1,2,3,4
$$

where $\Delta \mathrm{Q}=0.0039$ a.u.. The brown arrows indicate direction of increasing wave vectors. It should be noted that for $n=0$, the whole frequency range in Fig. 8 corresponds to the radiative region, but for all nonzero wave vectors $n>0$ (e.g., for $n=1$, $\mathrm{Qc}=14.5 \mathrm{eV})$, the whole frequency range corresponds to the evanescent region.

Figures 8(a)-8(c) show absorption spectra for $\mathrm{s}(x)$ polarized incident light for three different dopings $E_{F}=0,0.5$, and $1 \mathrm{eV}$, respectively. For pristine graphene $\left(E_{F}=0\right)$, far-IR absorption $(\omega<200 \mathrm{meV})$ slowly decreases as $\mathrm{Q}$ increases and shows positively dispersive peaks, with the most intense one for $n=1$ when it reaches almost $5 \%$ absorption. As Q increases, the intensities of these peaks decrease. The black dots in the Fig. 8(a) inset show the dispersion relation $\omega_{s}(\mathrm{Q})$ obtained by following the energies of these low-energy peaks
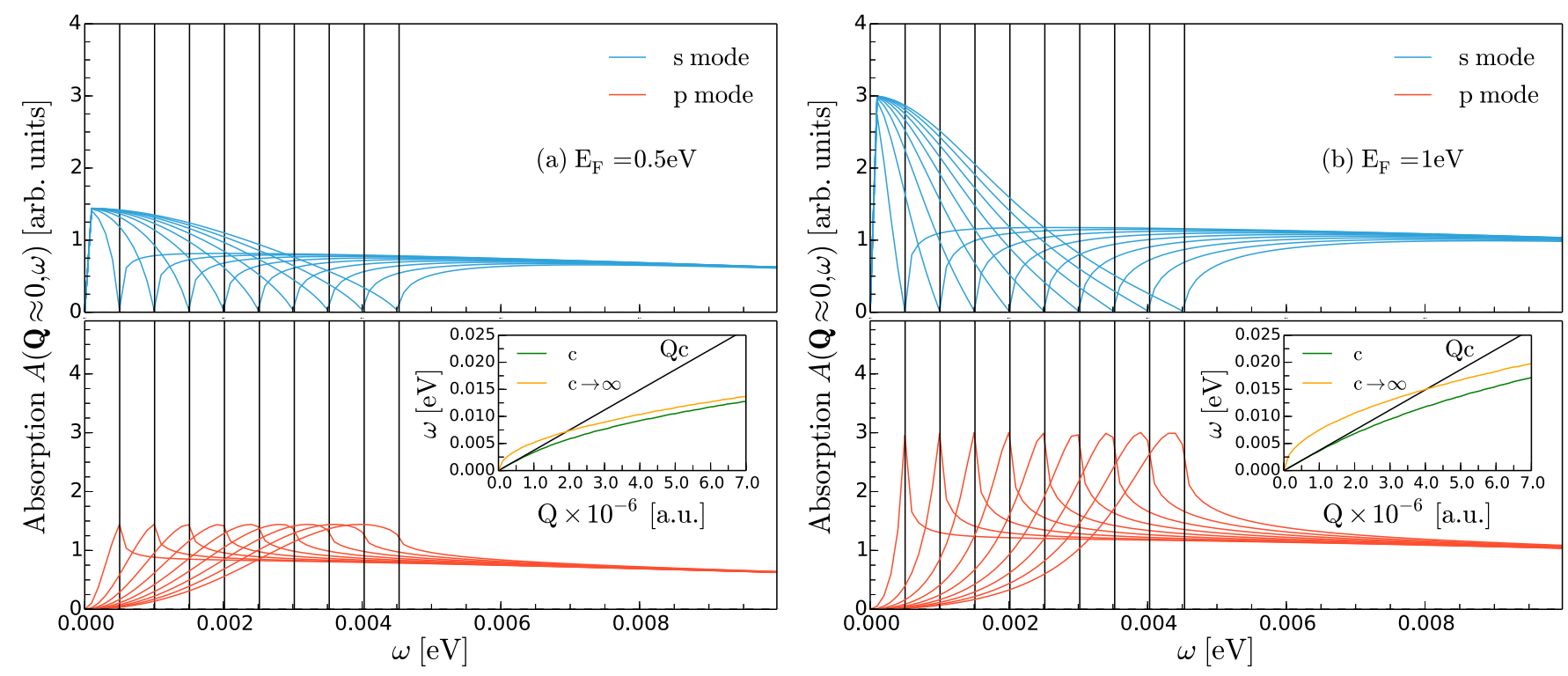

FIG. 6. Absorption spectrum for $\mathbf{s}$ (blue lines) and $\mathbf{p}$ (red lines) mode in doped graphene. Results are presented for (a) $E_{F}=0.5 \mathrm{eV}$ and (b) $E_{F}=1 \mathrm{eV}$. Incident wave vectors are in the $\Gamma M$ direction with the values $\mathbf{Q}_{n}=n \Delta \mathrm{Q} \hat{\mathbf{y}}, n=0-8$, where $\Delta \mathrm{Q}=1.35 \times 10^{-7}$ a.u. Vertical solid lines represent positions of the energy of light $\left(\omega_{\text {light }}=\mathrm{Qc}\right)$ for each of the wave vector Q. Insets: dispersion of the longitudinal $2 \mathrm{D}$ plasmon (TM mode) for each of the dopings, calculated when retardation effects are included ( $c$ is speed of light in the vacuum) (green line) and when retardation effects are not included $c \rightarrow \infty$ (yellow line). 

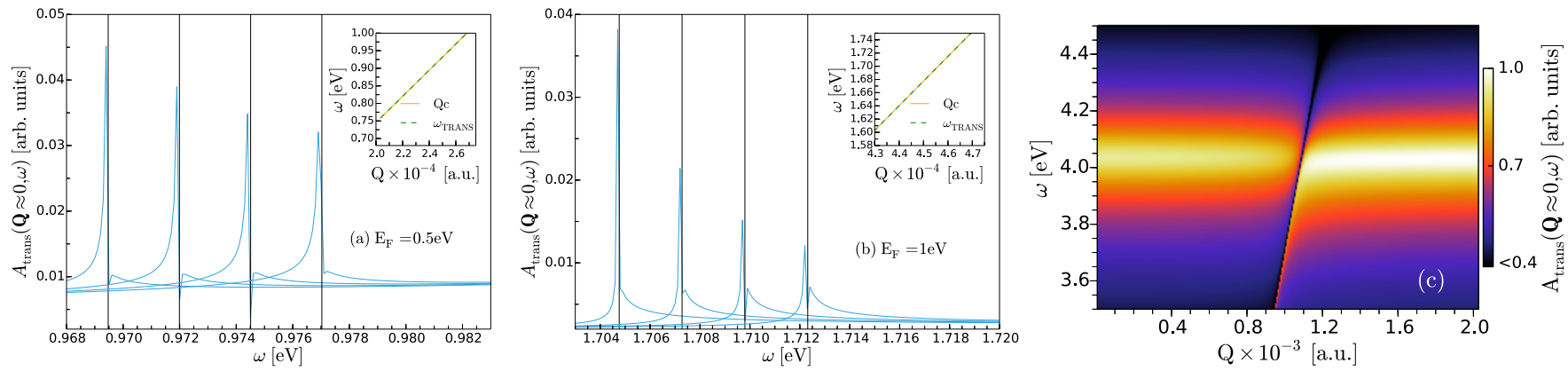

FIG. 7. (a) Absorption spectrum for $\mathbf{s}$ (blue lines) modes in doped graphene $\left(E_{F}=0.5 \mathrm{eV}\right)$ where incident wave vectors are $\mathbf{Q}_{n}=$ $\mathrm{Q}_{0}+n \Delta \mathrm{Q} \hat{\mathbf{y}}, n=0-3$, with $\mathrm{Q}_{0}=2.6 \times 10^{-4}$ a.u. and $\Delta \mathrm{Q}=6.76 \times 10^{-7}$ a.u. (b) Same as in (a) but with $E_{F}=1 \mathrm{eV}, \mathrm{Q}_{0}=4.6 \times 10^{-4}$ a.u., and $\Delta \mathrm{Q}=6.76 \times 10^{-7}$ a.u. Vertical solid lines are positions of the energy of light $\left(\omega_{\text {light }}=\mathrm{Qc}\right)$ for the corresponding wave vectors $\mathrm{Q}$. Insets: dispersion of the TE mode for each of the dopings compared with the dispersion of light. (c) Absorption intensities for $\mathbf{s}$ mode around the energies of $\pi \rightarrow \pi^{*}$ transitions for very small $\mathrm{Q}$ wave vectors $\left(\mathrm{Q} \sim \mathrm{Q}_{\text {light }}\right)$.

as functions of the wave vector $\mathrm{Q}$. We see that $\omega_{s}(\mathrm{Q})$ nicely follows the lower edge of the $\pi \rightarrow \pi^{*}$ interband continuum $\left(v_{F} \mathrm{Q}\right)$ for pristine graphene, shown by the black dashed line. The interband $\pi \rightarrow \pi^{*}$ peak at $\omega \approx 4 \mathrm{eV}$ remains dispersionless.

For doped graphene, the $\pi \rightarrow \pi$ intraband excitation channel is open and absorption spectra for $\mathrm{s}(x)$ polarized light get strong maxima in the far-IR region, as shown in Figs. 8(b) and 8(c). These absorption peaks are most intense for small Q's $(n=1,2)$ and rapidly decrease as $\mathrm{Q}$ increases.
The dispersion relations of these low-energy peaks $\omega_{s}(\mathrm{Q})$ are shown in the inset of Figs. 8(b) and 8(c). The upper edge of the intraband $\pi \rightarrow \pi$ electron-hole continuum is also shown (black dashed lines) for comparison. Around the Dirac cone, it can be approximated by $v_{F} \mathrm{Q}$, where for the Fermi velocity we took $v_{F}=\sqrt{3} t a / 2$ and where the hopping parameter of tight-binding model for graphene is $t \approx 2.7 \mathrm{eV}$ (as in [30]). We can notice that both peaks are linearly dispersive, where for $E_{F}=1 \mathrm{eV}, \omega_{s}(\mathrm{Q})$ follows exactly the $v_{F} \mathrm{Q}$ line. Because of the Pauli exclusion principle, as the doping increases the lower
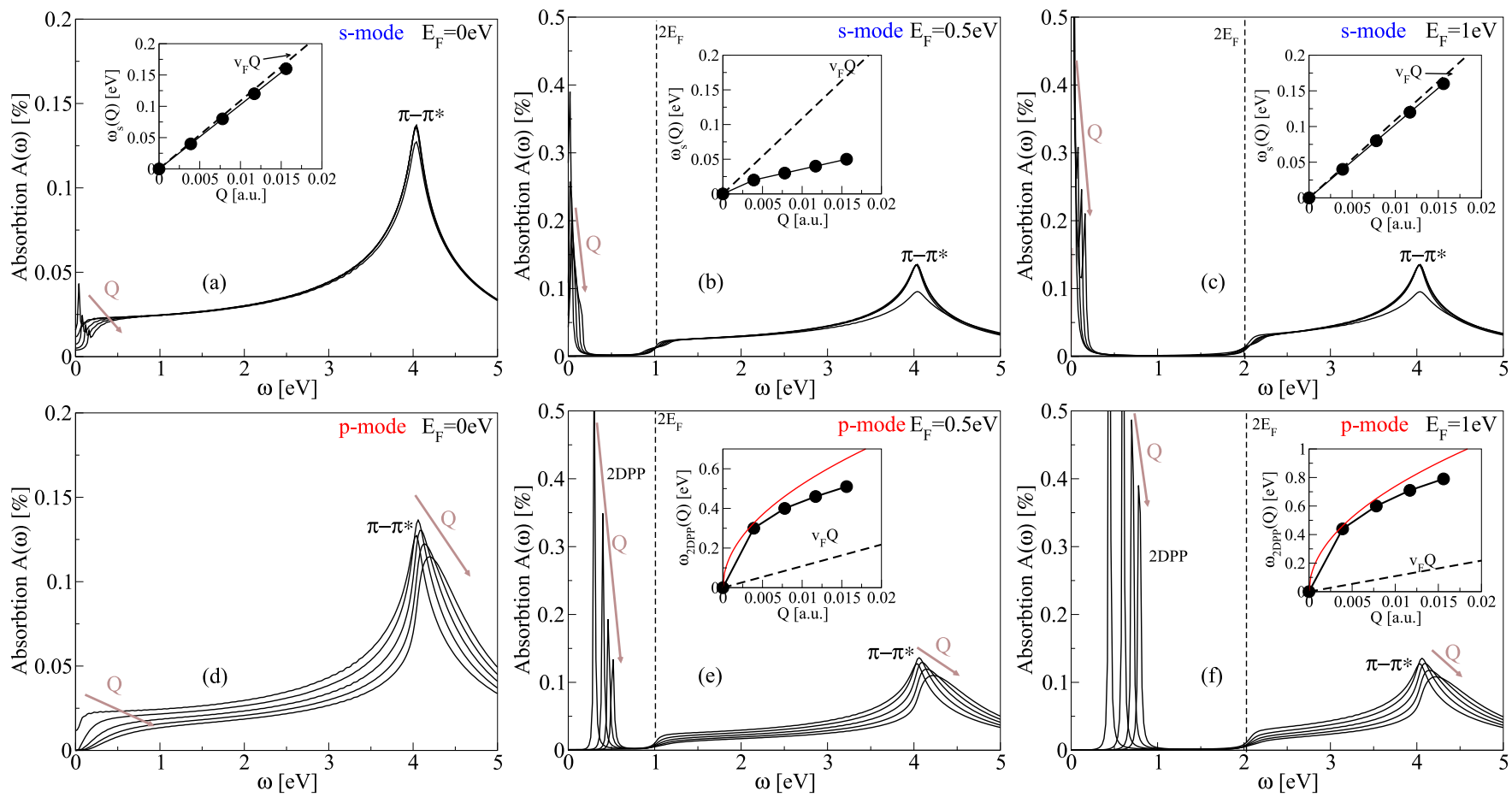

FIG. 8. Optical absorption spectrum for $\mathbf{s}$ and $\mathbf{p}$ polarized incident electromagnetic field in graphene with different doping: (a), (d) $E_{F}=0$; (b), (e) $E_{F}=0.5 \mathrm{eV}$; and (c), (f) $E_{F}=1 \mathrm{eV}$. In each of the graphs, the five absorption spectra are shown for five incident wave vectors along the $\Gamma \mathrm{M}$ direction $\mathbf{Q}_{n}=n \Delta \mathrm{Q} \mathbf{y}, n=0,1,2,3,4$, where $\Delta \mathrm{Q}=0.0039$ a.u. The brown arrows indicate the direction of increasing wave vector. Insets in (a), (b), and (c) show the dispersion relations of low-energy $\pi \rightarrow \pi$ intraband peaks, while insets in (e) and (f) show the dispersion relations of 2DPP. The dashed lines represent the upper edge $\left(v_{F} Q\right)$ of the $\pi \rightarrow \pi$ intraband electron-hole continuum where the Fermi velocity is $v_{F}=\sqrt{3} \mathrm{ta} / 2$ and the hopping parameter is $t=2.7 \mathrm{eV}$. Red solid lines represent the long-wavelength approximation of the 2DPP dispersion relation $\sqrt{2 E_{F} \mathrm{Q}}$. 
threshold for interband $\pi \rightarrow \pi^{*}$ transitions also increases. In other words, as these transitions are optically active, the increased doping will open the optical gap and move the absorption onset towards higher energies. In Figs. 8(b) and 8 (c), we can notice this wide optical gap in the whole IR and visible region (depending on the doping) with the optical absorption onset at $2 E_{F}$, denoted by the vertical dashed lines.

The absorption of $\mathrm{p}(y)$ polarized incident light is shown in Figs. 8(d)-8(f). The parallel wave vector $\mathbf{Q}$ is chosen also to be in the $y(\Gamma M)$ direction, so the response to this external electromagnetic field (for $Q \neq 0$ ) can be considered as longitudinal. It is evident from the results that this polarization gives generally more dispersive absorption spectrum in pristine graphene than the $\mathrm{s}(x)$ polarization. More specifically, we see that as $\mathrm{Q}$ increases, the value of $A(\omega \approx 0)$ rapidly decreases, and the $\pi \rightarrow \pi^{*}$ peak ( $\pi$ plasmon) becomes blue-shifted and less intense. In doped graphene, the Dirac cones are partially filled and q2D plasma is formed. As already mentioned, this plasma supports longitudinal self-sustaining oscillations called 2D plasmons [30], or in the electrodynamic limit, 2DPP. These 2DPP have evanescent character, i.e., electrical fields which they produce in the $z$ direction decay exponentially, as shown in Fig. 3(b). This implies that these modes exist in the $\omega<$ Qc region and cannot be excited (directly) by incident electromagnetic field which has fully radiative character. Figures 8(e) and 8(f) show absorption spectra of $\mathrm{p}(y)$ polarized incident field for doped graphene with $E_{F}=0.5$ and $1 \mathrm{eV}$, respectively. For $\mathrm{Q} \geqslant \Delta \mathrm{Q}$, the incident field has evanescent character in the shown frequency range and becomes capable to excite 2DPP, which gives rise to the strong peaks in the optical gap $v_{F} \mathrm{Q}<\omega<2 E_{F}$. Appearance of 2DPP causes strong screening which rapidly increases with $\mathrm{Q}$. One consequence of such screening is that for $\mathrm{p}(y)$ polarization, there is no intraband $\pi \rightarrow \pi$ absorption maximum in the far-IR region [as was the case for $\mathrm{s}(x)$ polarization]. The intensity of intraband $\pi \rightarrow \pi$ transitions is strongly reduced by the 2DPP screening field. The $2 \mathrm{DPP}$ dispersion relations $\omega_{2 \mathrm{DPP}}(\mathrm{Q})$ shown by black dots in the inset of Figs. 8(e) and 8(f) are compared with the simple long-wavelength approximation $\sqrt{2 E_{F} \mathrm{Q}}$ [30] (red solid line). The apparent disagreement between these two dispersion relations for $\omega>0.8 E_{F}$ is because in our calculations we considered both intraband and interband transitions, while to get the simplified dispersion one only accounts for the intraband transitions. In the region below $0.8 E_{F}$, the agreement is better because there the interband contributions to 2DPP dispersion are negligible [30,35,36].

The difference between absorption spectra of graphene, which here extends in the third dimension, and intrinsically 2D models is not very large (as shown in Ref. [30]). Namely, the $\pi \rightarrow \pi^{*}$ and $\pi \rightarrow \sigma^{*}$ transitions have in-plane character, i.e., the charge density (or current) oscillates parallel to the graphene plane. These transitions can be well modeled by purely 2D theories, such as the tight-binding (TBA) model $[35,36]$ where only inplane hopping is permitted, or by using a model of two 2D electron gases [65]. But, if one wants to investigate the out-of-plane optical response in q2D systems, the mentioned theories are no longer adequate, and our model which includes the third dimension enables such analysis. The out-of-plane dipole active modes can be an intriguing topic in q2D materials which are composed of two different
q2D materials. Carefully selected external polarization can excite such modes which can cause intersystem charge transfer important for photovoltaic devices.

\section{Optical conductivity}

In this section, we analyze optical conductivity obtained from (45) in the system exposed to the homogeneous electrical field directed in the $\mu=x$ or $y$ direction and the wave vector is $\mathrm{Q}=0$. For $\mathrm{Q}=0$, the response is completely transverse, $\mathrm{s}(x)$ and $\mathrm{p}(y)$ polarizations are equivalent, the screening is inactive, so the screened $\Pi$ and unscreened $\Pi^{0}$ current-current response tensors (51) become equal. As the crystal local field effects in the $z$ direction are negligible, only the $G_{z}=G_{z}^{\prime}=0$ component in $\Pi^{0}$ is nonzero. Also, because in the entire analyzed frequency region $\frac{\omega L}{c} \rightarrow 0$, the form factor in (41) becomes $F\left(G_{z}=0\right) \rightarrow 1$. Under these conditions, the absorption (42) becomes proportional to optical conductivity (45), i.e., $A(\omega) \sim \sigma_{\mu \mu}(\omega)$, and therefore the discussion of the optical conductivity corresponds to the discussion of absorption spectra which were thoroughly analyzed in Figs. 4 and 5 of the previous section. However, here we shall emphasize the Drude limit $(\omega \approx 0)$ and we focus on the case of pristine graphene only.

Figure 9 shows the interband, Eqs. (58) and (59), and intraband, Eq. (57), contributions to optical conductivity in pristine graphene. Our numerical method (being limited by finite-K point sampling) is not especially appropriate to study different limits (e.g., $T \rightarrow 0, \omega \rightarrow 0$, and $\eta \rightarrow 0$ ) when approaching the ballistic minimum conductivity, as explained in Refs. [66-68], but it can be very suitable to explore the

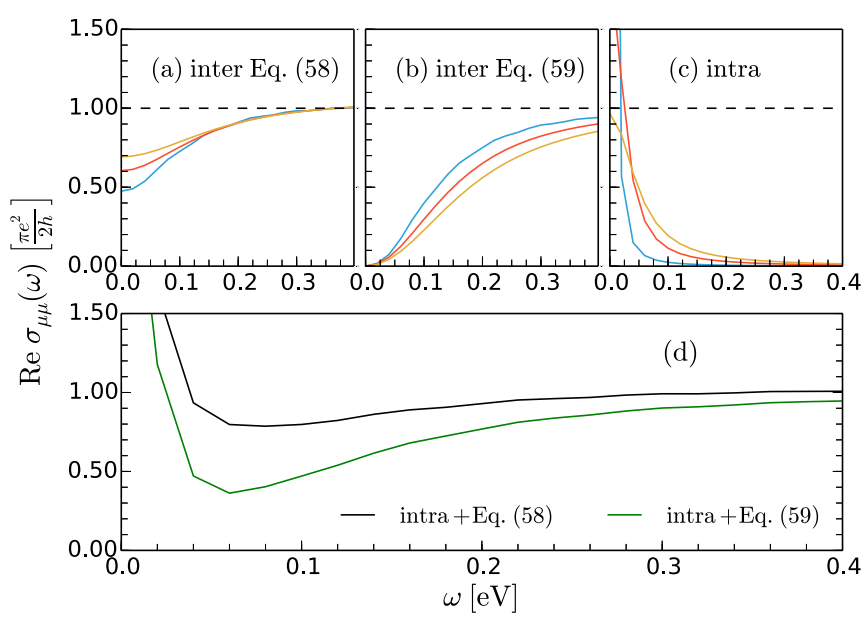

FIG. 9. (a) Real part of interband optical conductivity in pristine graphene $\left(E_{F}=0\right)$ calculated using Eq. (58) where the damping constants are $\eta_{\text {inter }}=60 \mathrm{meV}$ (blue), $\eta_{\text {inter }}=90 \mathrm{meV}$ (red), and $\eta_{\text {inter }}=120 \mathrm{meV}$ (yellow). (b) Same as in (a) but using Eq. (59). (c) Real part of intraband optical conductivity in pristine graphene ( $E_{F}=0$ ) calculated using Eq. (57) with $\eta_{\text {intra }}=5 \mathrm{meV}$ (blue), $\eta_{\text {intra }}=$ $25 \mathrm{meV}$ (red), and $\eta_{\text {intra }}=50 \mathrm{meV}$ (yellow), and $T=300 \mathrm{~K}$ for all three cases. (d) Total optical conductivity as a result of combining Eq. (57) with (58) (black) and (59) (green), where $\eta_{\text {intra }}=5 \mathrm{meV}$ and $\eta_{\text {inter }}=60 \mathrm{meV}$. The wave vector is $\mathrm{Q}=0$ which corresponds to the electromagnetic field of normal incidence and polarization $\mu=x$ or $y$. 
Drude regime. We use the finite temperature $(T=300 \mathrm{~K})$ and energy damping $(\eta>0)$ and in this case the intraband channel in pristine graphene is open [Fig. 9(c)]. We also present conductivity values for several damping rates $\eta$. Here, we see how the conductivity of graphene in Drude regime varies with the intensity of the relaxation processes [23]. Interband contributions to optical conductivity [Eqs. (58) and (59)] give two different behaviors for $\omega \gtrsim 0$ as seen in Figs. 9(a) and 9(b). The first gives finite values up to $\omega=0$, which is clearly an overestimation, while the second goes to 0 for $\omega=0$ as it should be in the case of graphene [22]. Now, it can be easily seen that Eq. (59) is more appropriate to use for the interband contribution to the optical conductivity at this level of approximation. Combining these two contributions [Eqs. (57) and (59)], one obtains the result which is in a good agreement with the previous work [5,18,21,22,24] [Fig. 9(d)].

\section{CONCLUSION}

In this paper, we presented a microscopic theory of electromagnetic response in q2D layered crystals, in terms of dynamically screened current-current response tensor. In this approach, the explicit knowledge of the electromagnetic field propagator can give us information about different polariton modes, both radiative and nonradiative, their spectra and intensities, their coupling to external fields and to other excitations in the crystal (e.g., phonons). Specifically, it is straightforward to evaluate optical properties of such crystals (absorption, reflection, transmission, and conductivity) and also to calculate higher-order many-body processes. The key physical quantity is the current-current response tensor, calculated from first principles which implies inclusion of the realistic crystal structure, wave functions, and electronic band structure. In order to test the developed formulation, we calculated optical absorption and conductivity in a singlelayer graphene and compared with recent experimental and theoretical results. The obtained results agree well with the measurements and experiments in IR and visible frequency regions, although the use of RPA is not capable to give correct excitonic effect. The developed theory is therefore suitable for study of dc or IR optical conductivity of conducting q2D crystals, such as, e.g., lithium (Li) or calcium (Ca) intercalated graphene which are nowadays of great importance in plasmonics. However, this theory is also capable to give insight in optical properties of semiconductiong q2D crystals such as, e.g., boron-nitride (BN), molibdenium-disulfide $\left(\mathrm{MoS}_{2}\right)$, or $\mathrm{MoS}_{2} /$ graphene composites nowadays important for photovoltaic devices.

\section{ACKNOWLEDGMENTS}

The authors are grateful to Donostia International Physics Center (DIPC) and P. M. Echenique for hospitality and financial support during various stages of this work. We also thank I. Kupčić and V. M. Silkin for useful discussions. Computation facilities were provided by the DIPC computing center.
[1] T. W. Ebbesen, H. J. Lezec, H. F. Ghaemi, T. Thio, and P. A. Wolff, Nature (London) 391, 667 (1998).

[2] D. Rossouw and G. A. Botton, Phys. Rev. Lett. 110, 066801 (2013).

[3] Hui-S. Kim, Jin-W. Lee, N. Yantara, P. P. Boix, S. A. Kulkarni, S. Mhaisalkar, M. Grätzel, and Nam-G. Park, Nano Lett. 13, 2412 (2013).

[4] F. J. Garcia-Vidal, L. Martin-Moreno, T. W. Ebbesen, and L. Kuipers, Rev. Mod. Phys. 82, 729 (2010).

[5] L. A. Falkovsky and A. A. Varlamov, Eur. Phys. J. B 56, 281 (2007).

[6] M. Jablan, H. Buljan, and M. Soljačić, Phys. Rev. B 80, 245435 (2009).

[7] G. Onida, L. Reining, and A. Rubio, Rev. Mod. Phys. 74, 601 (2002)

[8] T. Stauber and G. Gómez-Santos, New J. Phys. 14, 105018 (2012).

[9] A. Gutiérrez-Rubio, T. Stauber, and F. Guinea, J. Opt. 15, 114005 (2013).

[10] T. Stauber and G. Gómez-Santos, Phys. Rev. B 82, 155412 (2010).

[11] A. Principi, Marco Polini, and G. Vignale, Phys. Rev. B 80, 075418 (2009).

[12] R. D. King-Smith and J. C. Inkson, Phys. Rev. B 36, 4796 (1987).

[13] In principle, whether we use the charge-charge or current-current response function for calculating the polarizability of the system, we should end up with the same result (as required by the charge conservation and gauge invariance [45]), but in the numerical calculation this is not so easy to achieve due to the finite $k$-point sampling and the use of finite broadening parameter.

[14] V. Despoja, M. Šunjić, and L. Marušić, Phys. Rev. B 80, 075410 (2009).

[15] L. Yang, J. Deslippe, C.-H. Park, M. L. Cohen, and S. G. Louie, Phys. Rev. Lett. 103, 186802 (2009).

[16] Kin Fai Mak, Long Ju, Feng Wang, and Tony F. Heinz, Solid State Commun. 152, 1341 (2012).

[17] R. R. Nair, P. Blake, A. N. Grigorenko, K. S. Novoselov, T. J. Booth, T. Stauber, N. M. R. Peres, and A. K. Geim, Science 320, 1308 (2008).

[18] Kin Fai Mak, Matthew Y. Sfeir, Yang Wu, Chun Hung Lui, James A. Misewich, and Tony F. Heinz, Phys. Rev. Lett. 101, 196405 (2008).

[19] K. S. Novoselov, A. K. Geim, S. V. Morozov, D. Jiang, Y. Zhang, S. V. Dubonos, I. V. Grigorieva, and A. A. Firsov, Science 306, 666 (2004).

[20] K. I. Bolotin, K. J. Sikes, J. Hone, H. L. Stormer, and P. Kim, Phys. Rev. Lett. 101, 096802 (2008).

[21] A. B. Kuzmenko, E. van Heumen, F. Carbone, and D. van der Marel, Phys. Rev. Lett. 100, 117401 (2008).

[22] T. Stauber, N. M. R. Peres, and A. K. Geim, Phys. Rev. B 78, 085432 (2008). 
[23] N. M. R. Peres, F. Guinea, and A. H. Castro Neto, Phys. Rev. B 73, 125411 (2006).

[24] V. P. Gusynin, S. G. Sharapov, and J. P. Carbotte, Phys. Rev. Lett. 96, 256802 (2006).

[25] A. G. Marinopoulos, L. Reining, A. Rubio, and V. Olevano, Phys. Rev. B 69, 245419 (2004).

[26] S. C. Liou, C.-S. Shie, C. H. Chen, R. Breitwieser, W. W. Pai, G. Y. Guo, and M.-W. Chu, Phys. Rev. B 91, 045418 (2015).

[27] P. Wachsmuth, R. Hambach, M. K. Kinyanjui, M. Guzzo, G. Benner, and U. Kaiser, Phys. Rev. B 88, 075433 (2013).

[28] P. Wachsmuth, R. Hambach, G. Benner, and U. Kaiser, Phys. Rev. B 90, 235434 (2014).

[29] F. J. Nelson, J.-C. Idrobo, J. D. Fite, Z. L. Mišković, S. J. Pennycook, S. T. Pantelides, J. U. Lee, and A. C. Diebold, Nano Lett. 14, 3827 (2014).

[30] V. Despoja, D. Novko, K. Dekanić, M. Šunjić, and L. Marušić, Phys. Rev. B 87, 075447 (2013).

[31] D. Novko, V. Despoja, and M. Šunjić, Phys. Rev. B 91, 195407 (2015).

[32] T. Low and P. Avouris, ACS Nano 8, 1086 (2014).

[33] E. H. Hwang and S. Das Sarma, Phys. Rev. B 75, 205418 (2007).

[34] M. Pisarra, A. Sindona, P. Riccardi, V. M. Silkin, and J. M. Pitarke, New J. Phys. 16, 083003 (2014).

[35] I. Kupčić, Phys. Rev. B 90, 205426 (2014).

[36] I. Kupčić, Phys. Rev. B 91, 205428 (2015).

[37] S. A. Mikhailov and K. Ziegler, Phys. Rev. Lett. 99, 016803 (2007).

[38] Marinko Jablan, Hrvoje Buljan, and Marin Soljačić, Opt. Express 19, 11236 (2011).

[39] F. M. D. Pellegrino, G. G. N. Angilella, and R. Pucci, Phys. Rev. B 84, 195407 (2011).

[40] Xu Du, Ivan Skachko, Anthony Barker, and Eva Y. Andrei, Nat. Nanotechnol. 3, 491 (2008).

[41] V. U. Nazarov, New J. Phys. 17, 073018 (2015).

[42] V. U. Nazarov, Phys. Rev. B 92, 161402(R) (2015).

[43] A. Politano, G. Chiarello, and A. Cupolillo, New J. Phys. 17, 081002 (2015).

[44] Thibault Sohier, Matteo Calandra, and Francesco Mauri, Phys. Rev. B 91, 165428 (2015).

[45] J. R. Schrieffer, Theory of Superconductivity (Benjamin, New York, 1964).

[46] Thomas Olsen and Kristian S. Thygesen, Phys. Rev. B 87, 075111 (2013).
[47] M. S. Tomaš, Phys. Rev. A 51, 2545 (1995).

[48] V. Despoja, I. Lončarić, D. J. Mowbray, and L. Marušić, Phys. Rev. B 88, 235437 (2013).

[49] I. Kupčić, Phys. B (Amsterdam) 322, 154 (2002).

[50] J. M. Ziman, Principles of the Theory of Solids (Cambridge University Press, London, 1979).

[51] F. Wooten, Optical Properties of Solids (Academic, New York, 1972).

[52] N. W. Ashcroft and K. Sturm, Phys. Rev. B 3, 1898 (1971).

[53] The informal derivation of Eq. (59) can be done by expressing Eq. (58) separately in $f_{n \mathbf{K}}$ and $f_{m \mathbf{K}}$ parts, then switching the dummy variables in the second part $n \leftrightarrow m$ and finally combining them together with the crucial step of removing $i \eta_{\text {inter }}$ from the numerator. This parameter in the numerator is responsible for the spurious behavior of Eq. (58). Now, we can see that the difference between Eqs. (58) and (59) is the factor $\hbar \omega /\left(\hbar \omega+i \eta_{\text {inter }}\right)$. Sometimes, it is a custom to disregard also $\eta_{\text {inter }}^{2}$ in the denominator [50,51].

[54] V. Despoja, M. Šunjić, and L. Marušić, Phys. Rev. B 83, 165421 (2011).

[55] G. D. Mahan, Many-Particle Physics, 2nd ed. (Pergamon, New York, 1990).

[56] R. Saito, G. Dresselhaus, and M. S. Dresselhaus, Physical Properties of Carbon Nanotubes (Imperial College Press, London, 1998).

[57] P. Giannozzi, S. Baroni, N. Bonini, M. Calandra, R. Car, C. Cavazzoni, D. Ceresoli, G. L. Chiarotti, M. Cococcioni, I. Dabo et al., J. Phys.: Condens. Matter 21, 395502 (2009).

[58] N. Troullier and J. L. Martins, Phys. Rev. B 43, 1993 (1991).

[59] J. P. Perdew and A. Zunger, Phys. Rev. B 23, 5048 (1981).

[60] H. J. Monkhorst and J. D. Pack, Phys. Rev. B 13, 5188 (1976).

[61] V. M. Silkin, E. V. Chulkov, and P. M. Echenique, Phys. Rev. Lett. 93, 176801 (2004).

[62] F. M. D. Pellegrino, G. G. N. Angilella, and R. Pucci, Phys. Rev. B 82, 115434 (2010).

[63] F. M. D. Pellegrino, G. G. N. Angilella, and R. Pucci, High Press. Res. 31, 98 (2011).

[64] F. Stern, Phys. Rev. Lett. 18, 546 (1967).

[65] V. B. Jovanović, I. Radović, D. Borka, and Z. L. Mišković, Phys. Rev. B 84, 155416 (2011).

[66] K. Ziegler, Phys. Rev. Lett. 97, 266802 (2006).

[67] K. Ziegler, Phys. Rev. B 75, 233407 (2007).

[68] S. Das Sarma, Shaffique Adam, E. H. Hwang, and Enrico Rossi, Rev. Mod. Phys. 83, 407 (2011). 